\title{
Gauged Supergravities in Three Dimensions: A Panoramic Overview
}

\section{B. de Wit*}

Institute for Theoretical Physics $\&$ Spinoza Institute, Utrecht University, Postbus 80.195, 3508 TD Utrecht, The Netherlands

E-mail: 'b.dewit@phys.uu.n̄'

\section{H. Nicolai ${ }^{\dagger}$}

\author{
Max-Planck-Institut für Gravitationsphysik, \\ Albert-Einstein-Institut, \\ Mühlenberg 1, D-14476 Potsdam, Germany \\ E-mail: 'nicolai@aei mpg. de',
}

\section{H. Samtleben}

II. Institut für Theoretische Physik der Universität Hamburg, Luruper Chaussee 149, D-22761 Hamburg, Germany

E-mail: henning. samtleben@desy.de'

ABSTRACT: Maximal and non-maximal supergravities in three spacetime dimensions allow for a large variety of semisimple and non-semisimple gauge groups, as well as complex gauge groups that have no analog in higher dimensions. In this contribution we review the recent progress in constructing these theories and discuss some of their possible applications.

\section{Introduction}

Locally supersymmetric theories in three spacetime dimensions coupled to matter have at most $N=16$ supersymmetries [i]1] which parametrize a target space belonging to a nonlinear sigma model. While there is a large number of possible target spaces when $N \leq 4$, the possibilities become more restricted with increasing $N$ : beyond $N=4$, the target spaces are coset spaces $\mathrm{G} / \mathrm{H}$, where $\mathrm{H}$ is the maximal compact subgroup of $\mathrm{G}$. For all values of $N$ these supergravities may be invariant under a bosonic symmetry group $\mathrm{G}$, which commutes with the Lorentz

\footnotetext{
${ }^{*}$ Speaker.

${ }^{\dagger}$ Speaker.
} 
transformations and spacetime diffeomorphisms and which involves (subgroups of) the target-space isometry group and the R-symmetry group $\mathrm{SO}(N)$. In that case there exist supersymmetric deformations where a subgroup $\mathrm{G}_{0} \subset \mathrm{G}$ is promoted to a local symmetry

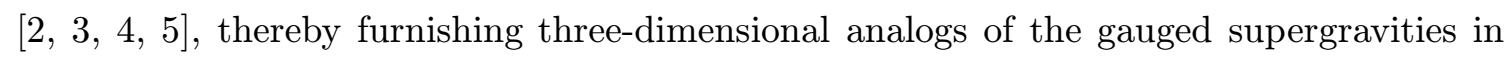
dimensions $D \geq 4$ that have been known for a long time. In contrast to higher-dimensional gauged supergravities, the vector fields in general appear via a Chern-Simons (CS) rather than a Yang-Mills (YM) term. As it turns out, there is a surprisingly rich structure and variety of possible gaugings, including semisimple and non-semisimple gauge groups as well as novel complex gaugings which have no analog in $D \geq 4$ dimensions.

There are several reasons why $D=3$ (gauged) supergravities are of more general interest. Below we list some of these reasons.

- During the last five years there has been enormous interest in the so-called AdS/CFT correspondence, according to which a supergravity theory with an AdS groundstate is related to a (super)conformal theory living on the boundary of the AdS space (see

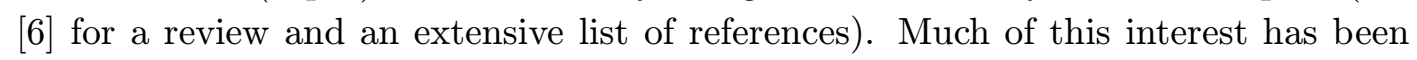
focused on the $\mathrm{AdS}_{5} / \mathrm{CFT}_{4}$ correspondence, relating gauged maximal supergravity with gauge group $\mathrm{SO}(6)$ on $\mathrm{AdS}_{5}[\bar{i}]$ ] to the maximally supersymmetric Yang-Mills theory on its boundary. While in this case one has essentially only one theory to test the conjectured correspondence, the number of possibilities is far greater when one descends by two in the number of dimensions: the $\mathrm{AdS}_{3} / \mathrm{CFT}_{2}$ correspondence offers a much larger bestiary of examples, because on the one hand there are far more superconformal theories in two dimensions, and on the other hand because gauged supergravities are more numerous in three dimensions.

- (Ungauged) extended supergravities exhibit their maximal global and most "unified" symmetry in three dimensions ${ }^{1}$, because all tensor gauge fields can be dualized to scalar fields, so that the propagating bosonic degrees of freedom are uniformly described by scalar fields, which usually live on a target space with a nice geometrical structure. In particular for the maximal $N=16$ theory, the maximally extended

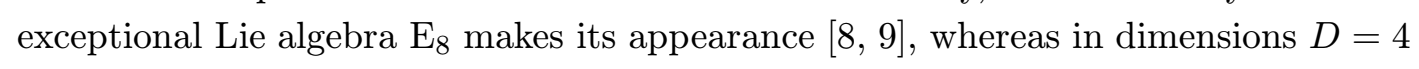
and $D=5$ the maximal-rank exceptional symmetries compatible with maximal supersymmetry are $\mathrm{E}_{7}$ and $\mathrm{E}_{6}$, respectively [i $\left.{ }^{1} \bar{Q}_{1}^{\prime}\right]$.

- Unlike the abelian duality relating scalar fields and antisymmetric tensor gauge fields in higher dimensions, the duality between scalar and vector fields can be extended to non-abelian gauge groups in three dimensions. There is a novel equivalence between YM and certain CS gauge theories (which also holds for non-supersymmetric theories) which has no analog in dimensions $D \geq 4$. Namely, as shown in $\left[\begin{array}{c}1 \\ 1\end{array}\right.$ dimensions, any $Y M$ gauged supergravity with gauge group $G_{Y M}$ is equivalent to a CS gauged supergravity with non-semisimple gauge group $\mathrm{G}_{\mathrm{YM}} \ltimes \mathcal{T}$ with a certain translation group $\mathcal{T}$. Because in the latter formulation all the vectors appear via a

\footnotetext{
${ }^{1}$ Here we will not be concerned with the infinite dimensional global symmetries $E_{9}, E_{10}$ and $E_{11}$, which are known, resp. conjectured, to emerge for maximal supergravities in dimensions $D \leq 2$.
} 
CS term rather than a YM-type kinetic term, no new propagating degrees of freedom are generated by the gauging, as is required by the preservation of supersymmetry. Altogether, the CS gauged supergravities thus not only contain the YM-type gauged theories but encompass a much larger class of theories.

- Because the vectors appear via a CS term and do not propagate, their number and hence the dimension of the gauge group are not determined a priori, unlike in dimensions $D \geq 4$. For this reason, the possible gaugings are more numerous and exhibit a richer structure than the corresponding $D \geq 4$ gauged supergravities. Similar comments apply to the scalar potentials of these theories which provide a large variety of symmetry breaking patterns with vacua of the anti-de Sitter, Minkowski or de Sitter-

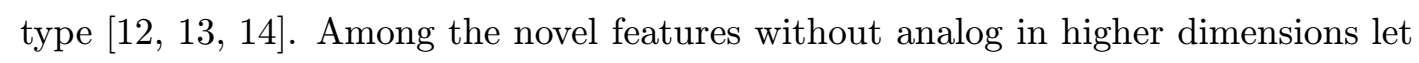
us mention the existence of maximally supersymmetric vacua for non-compact gauge groups (cf. table 2 in section 9) and the occurrence of stable AdS-type vacua with completely broken supersymmetry (for $D \geq 4$, all known non-supersymmetric vacua

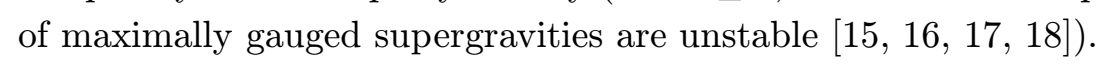

- Except for certain non-semisimple gaugings, none of the $D=3$ gauged supergravities can be obtained by any known mechanism from higher dimensional supergravity. The very existence of these theories may thus point to the existence of new "cusps" of $\mathrm{M}$ theory, and the existence of new geometrical structures in eleven dimensions of the type suggested in $\left[\underline{1}_{1}^{\overline{1}} \bar{g}_{1}, \overline{2} \overline{\underline{Q}}\right]$ - $]$ and references therein. The theories which do originate from higher dimensions usually appear with a YM-type kinetic term, and therefore necessarily require non-semisimple gauge groups in the CS-type formulation, as described above. In particular they include all those theories obtained by reduction of higher-dimensional maximal gauged supergravities on a torus, or by Kaluza-Klein compactification of higher-dimensional supergravities on some internal manifold, such as for instance IIA/IIB supergravity compactified on the seven-sphere, or $D=5$ supergravity on the two-sphere.

- Just like $D=11$ supergravity can be viewed as a strong-coupling limit of $D=10$ IIA superstring theory [2핌] one may speculate that four dimensions might arise out of a strongly coupled $D=3$ supergravity theory [i2 $2 \overline{2}]$. In this context, a special role is played by the dilaton field, whose expectation value on the one hand 'measures' the size of the $S^{1}$ on which one reduces, and on the other hand appears as the string coupling constant. The connection between the pertinent $D=3$ potentials and the potentials of $D \geq 4$ gauged supergravity potentials has been studied in $\left[\begin{array}{l}4 \\ 0\end{array}\right]$, where the dilaton is identified with the scalar field associated with a certain grading operator which is an element of the relevant (non-semisimple) gauge group.

- Gauged supergravity can provide an effective and economical description of an infinite number of Kaluza-Klein supermultiplets in a way that is again without analog in dimensions $D \geq 4$. This has been recently demonstrated for the compactification of matter-coupled half maximal $D=6$ supergravity on $\operatorname{AdS}_{3} \times S^{3}$ which leads to an 
effective theory in three dimensions with $N=8$ local supersymmetries [i2 $2 \overline{3}]$. More specifically, the self-interactions of the the spin-1 Kaluza-Klein towers are fully described by an $N=8$ gauged supergravity with gauge group $\mathrm{SO}(4) \ltimes \mathcal{T}_{\infty}$, where $\mathcal{T}_{\infty}$ is an infinite dimensional translation group, and the gauge group is embedded into the global symmetry group $\mathrm{SO}(8, \infty)$. (The second entry is infinite because there are infinitely many $N=8$ matter supermultiplets.) In particular, this embedding is compatible with the quantum numbers of the Kaluza-Klein supermultiplets, and the masses of all Kaluza-Klein states are correctly recovered from a single scalar potential.

- Finally, there are intriguing connections to recent developments in the differential geometry of three-dimensional manifolds. On the one hand, the models contain the CS Lagrangians that can be used to describe knots and links and their characteristic

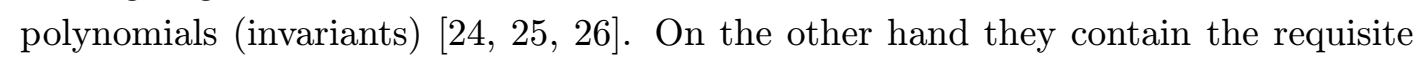
matter fields to realize the various elementary Thurston geometries [2] $\left.\overline{2}_{1} \bar{Z}_{1}, \overline{2} \overline{2} \overline{8}\right]$; in particular, recent progress in establishing part of the Thurston conjecture [20 $\overline{2}]$ ] has been based on the introduction of a 'dilaton field'. The question is therefore whether these gauged supergravities can provide a unified framework for these so far disconnected parts of mathematics.

This review is organized as follows. In section 2 we briefly review the results of [i] on the ungauged supergravity theories in three dimensions. The global invariances of the corresponding Lagrangians are discussed in section 3. In section 4 we show that arbitrary gauge field couplings of Yang-Mills-type in three dimensions may always be brought into the form of particular Chern-Simons interactions. For general gaugings we may thus restrict attention to the latter type of theories. In section 5 and 6 we present the full Lagrangian and transformation rules of the gauged supergravities in three dimensions, as well as the conditions that must be satisfied in order that the gauging preserves supersymmetry. The theories for $N \leq 4$ supersymmetries are discussed in more detail in section 7 , while sections 8 and 9 focus on the structure of the $N>4$ theories, and in particular on the admissible gauge groups for the maximal $(N=16)$ theory. There, we also mention some possible implications of our results for the $\mathrm{AdS}_{3} / \mathrm{CFT}_{2}$ correspondence.

\section{Supergravity coupled to nonlinear sigma models}

In this section we briefly summarize the results of [i] (for a discussion of the peculiarities

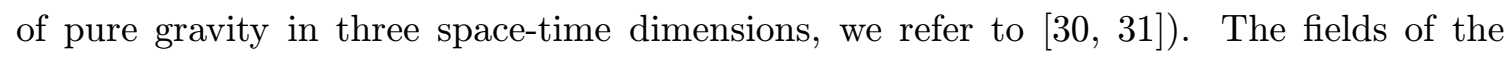
nonlinear sigma model are the target-space coordinates $\phi^{i}$ and their superpartners $\chi^{i}$, with $i=1, \ldots, d$; the supergravity fields are the dreibein $e_{\mu}{ }^{a}$, the spin-connection field $\omega_{\mu}{ }^{a b}$ and $N$ gravitino fields $\psi_{\mu}^{I}$ with $I=1, \ldots, N$. The gravitinos transform under the R-symmetry group $\mathrm{SO}(N)$, which is not necessarily a symmetry group of the Lagrangian.

Since the fields are all massless at this stage, one may assume that no matter fields other than scalars and spinors are required, because helicity is trivial in three dimensions. The scalar fields parametrize a target space endowed with a Riemannian metric $g_{i j}(\phi)$. 
Pure supergravity is topological in three dimensions and exists for an arbitrary number $N$ of supercharges and corresponding gravitinos [i] $\left.{ }^{2} \overline{2}\right]$. Its coupling to a nonlinear sigma model requires the existence of $N-1$ hermitean, almost complex, structures $f^{P i}{ }_{j}(\phi)$, labeled by $P=2, \ldots, N$, which generate a Clifford algebra,

$$
f_{k}^{P i} f^{Q k}{ }_{j}+f_{k}^{Q i} f_{j}^{P k}=-2 \delta^{P Q} \delta_{j}^{i} .
$$

From the $f^{P}$ one constructs $\frac{1}{2} N(N-1)$ tensors $f_{i j}^{I J}=-f_{i j}^{J I}=-f_{j i}^{I J}$ that act as generators for the group $\mathrm{SO}(N)$,

$$
f^{P Q}=f^{[P} f^{Q]}, \quad f^{1 P}=-f^{P 1}=f^{P},
$$

where, here and henceforth, $I, J=1, \ldots, N$. The $f^{I J}$ are covariantly constant, both with respect to the Christoffel and $\mathrm{SO}(N)$ target-space connections, $\Gamma_{i j}{ }^{k}$ and $Q_{i}^{I J}$, respectively,

$$
D_{i}(\Gamma, Q) f_{j k}^{I J} \equiv \partial_{i} f_{j k}^{I J}-2 \Gamma_{i[k}^{l} f_{j] l}^{I J}+2 Q_{i}^{K[I} f_{j k}^{J] K}=0 .
$$

The $\mathrm{SO}(N)$ connections $Q_{i}^{I J}(\phi)$ are nontrivial in view of

$$
R_{i j}^{I J}(Q) \equiv \partial_{i} Q_{j}^{I J}-\partial_{j} Q_{i}^{I J}+2 Q_{i}^{K[I} Q_{j}^{J] K}=\frac{1}{2} f_{i j}^{I J} .
$$

For $N=2$ the target space is Kähler and $f^{12}$ is a complex structure. The $\mathrm{SO}(2)$ holonomy is undetermined. For $N=3$, there are three (almost) complex structures $f^{12}, f^{23}$ and $f^{31}$, and the target space is a quaternion-Kähler space. The case $N=4$ is special: there exists a tensor $J^{i}{ }_{j}$, defined by

$$
J=\frac{1}{24} \varepsilon^{I J K L} f^{I J} f^{K L}, \quad J^{2}=\mathbf{1},
$$

which has eigenvalues \pm 1 , commutes with the almost complex structures and is covariantly constant. This implies that the target space is locally the product of two separate Riemannian spaces of dimension $d_{ \pm}$, where $d_{+}+d_{-}=d$ and $d_{ \pm}$are both multiples of 4 . These two subspaces are quaternion-Kähler and correspond to inequivalent $N=4$ supermultiplets. For $N=4$ we note the following identity,

$$
f^{I J i j} f^{K L}{ }_{i j}=4\left(d_{+} \mathbb{P}_{+}^{I J, K L}+d_{-} \mathbb{P}_{-}^{I J, K L}\right),
$$

with (anti)self-dual projectors,

$$
\mathbb{P}_{ \pm}^{I J, K L}=\frac{1}{2} \delta^{I[K} \delta^{L] J} \mp \frac{1}{4} \varepsilon^{I J K L}
$$

For $N>2$ the target space is an Einstein space with nontrivial $\mathrm{SO}(N)$ holonomy. The holonomy group is contained in $\mathrm{SO}(N) \times \mathrm{H}^{\prime} \subset \mathrm{SO}(d)$ which must act irreducibly on the target space. The group $\mathrm{H}^{\prime}$ must be a subgroup of $\mathrm{SO}(k)$ (for $\left.N=7,8,9 \bmod 8\right), \mathrm{U}(k)$ (for $N=2,6 \bmod 8$ ), or $\operatorname{Sp}(k)$ (for $N=3,4,5 \bmod 8$ ), where $k$ denotes the number of independent supermultiplets whose scalar fields parametrize the target space. For $N=4$ 


\begin{tabular}{||c|c|c||}
\hline$N$ & Target Space & $d$ \\
\hline \hline 1 & Riemann manifold $\mathcal{M}_{\mathrm{R}}$ & $k$ \\
\hline 2 & Kähler manifold $\mathcal{M}_{\mathrm{K}}$ & $2 k$ \\
\hline 3 & quaternion Kähler manifold $\mathcal{M}_{\mathrm{QK}}$ & $4 k$ \\
\hline 4 & quaternion Kähler manifolds $\mathcal{M}_{\mathrm{QK} 1} \times \mathcal{M}_{\mathrm{QK} 2}$ & $4\left(k_{1}+k_{2}\right)$ \\
\hline 5 & $\mathrm{Sp}(2, k) /(\mathrm{Sp}(2) \times \mathrm{Sp}(k))$ & $8 k$ \\
\hline 6 & $\mathrm{SU}(4, k) /(\mathrm{SU}(k) \times \mathrm{SU}(4) \times \mathrm{U}(1))$ & $8 k$ \\
\hline 8 & $\mathrm{SO}(8, k) /(\mathrm{SO}(8) \times \mathrm{SO}(k))$ & $8 k$ \\
\hline 9 & $\mathrm{~F}_{4(-20)} / \mathrm{SO}(9)$ & 16 \\
\hline 10 & $\mathrm{E}_{6(-14)} /(\mathrm{SO}(10) \times \mathrm{U}(1))$ & 32 \\
\hline 12 & $\mathrm{E}_{7(-5)} /(\mathrm{SO}(12) \times \mathrm{Sp}(1))$ & 64 \\
\hline 16 & $\mathrm{E}_{8(8)} / \mathrm{SO}(16)$ & 128 \\
\hline
\end{tabular}

Table 1: Target spaces for $D=3$ supergravities. The number of independent supermultiplets is denoted by $k$. For $N=4$ there exist two types of (inequivalent) supermultiplets, counted by $k_{1}$ and $k_{2}$.

these results are more subtle because of the product structure. We note the following relations (always assuming $N>2$ ),

$$
\begin{aligned}
R_{i j k l} & =\frac{1}{8}\left(f_{i j}^{I J} f_{k l}^{I J}+C_{\alpha \beta} h_{i j}^{\alpha} h_{k l}^{\beta}\right), \\
R_{i j k l} f^{I J k l} & =\frac{1}{2}\left(d_{+} \mathbb{P}_{+}{ }^{I J, K L}+d_{-} \mathbb{P}_{-}^{I J, K L}\right) f_{i j}^{K L}, \\
R_{i j} & =\left(N-2+\frac{1}{8} d\right) g_{i j}+\frac{1}{8}\left(d_{+}-d_{-}\right) J_{i j},
\end{aligned}
$$

where, for $N \neq 4$, one must set $J_{i j}=0$ and $\mathbb{P}_{ \pm}^{I J, K L}=\frac{1}{2} \delta^{I[K} \delta^{L] J}$. In the first equation, $C_{\alpha \beta}(\phi)$ is a symmetric tensor and the target-space tensors $h_{i j}^{\alpha}(\phi)$ form a basis of antisymmetric tensors commuting with the almost complex structures. These tensors generate the $\mathrm{H}^{\prime}$ factor of the holonomy group with corresponding structure constants $f^{\alpha \beta}$.

Beyond $N=4$ the target space geometries become very restricted. This is shown in table $i_{-1}^{1}$, where $k$ denotes the number of matter supermultiplets coupled to supergravity. Remarkably, not all values of $4<N \leq 16$ can be realized: matter-coupled supergravities exist only for $N=5,6,8,9,10,12$ and 16 supercharges. Furthermore, only for $N \leq 8$ is it possible to include an arbitrary number $k$ of supermultiplets, whereas for $N \geq 9$ there exists only one theory for each admissible value of $N$.

Let us now turn to the Lagrangian and supersymmetry transformations. We adopt a manifestly $\mathrm{SO}(N)$ covariant notation which allows to select the $N-1$ almost complex structures from the $f^{I J}$ tensors by specifying some arbitrary unit $N$-vector $\alpha_{I}$ and identifying the complex structures with $\alpha_{J} f^{J I}$. Accordingly we extend the fermion fields $\chi^{i}$ to 
an overcomplete set, $\chi^{i I}$, defined by

$$
\chi^{i I}=\left(\chi^{i}, f^{P i} \chi^{j}\right) .
$$

The fact that we have only $d$ fermion fields, rather than $d N$, is expressed by the $\mathrm{SO}(N)$ covariant constraint,

$$
\chi^{i I}=\mathbb{P}_{J j}^{I i} \chi^{j J} \equiv \frac{1}{N}\left(\delta^{I J} \delta_{j}^{i}-f_{j}^{I J i}\right) \chi^{j J}
$$

We should stress, that the introduction of $\chi^{i I}$ is a purely notational convenience; at every step in the computation one may change back to the original notation by choosing $\chi^{i}=$ $\alpha_{I} \chi^{i I}$. The covariant notation does not imply that the theory is $\mathrm{SO}(N)$ invariant; rather the covariant setting allows us to treat the $N$ supersymmetries and the corresponding gravitinos on equal footing.

The Lagrangian then takes the form

$$
\begin{aligned}
\mathcal{L}_{0}= & -\frac{1}{2} \mathrm{i} \varepsilon^{\mu \nu \rho}\left(e_{\mu}{ }^{a} R_{\nu \rho a}+\bar{\psi}_{\mu}^{I} D_{\nu} \psi_{\rho}^{I}\right)-\frac{1}{2} e g_{i j}\left(g^{\mu \nu} \partial_{\mu} \phi^{i} \partial_{\nu} \phi^{j}+N^{-1} \bar{\chi}^{i I} \not D \chi^{j I}\right) \\
& +\frac{1}{4} e g_{i j} \bar{\chi}^{i I} \gamma^{\mu} \gamma^{\nu} \psi_{\mu}^{I}\left(\partial_{\nu} \phi^{j}+\widehat{\partial}_{\nu} \phi^{j}\right)-\frac{1}{24} e N^{-2} R_{i j k l} \bar{\chi}^{i I} \gamma_{a} \chi^{j I} \bar{\chi}^{k J} \gamma^{a} \chi^{l J} \\
& +\frac{1}{48} e N^{-2}\left(3\left(g_{i j} \bar{\chi}^{i I} \chi^{j I}\right)^{2}-2(N-2)\left(g_{i j} \bar{\chi}^{i I} \gamma^{a} \chi^{j J}\right)^{2}\right)
\end{aligned}
$$

with the covariant derivatives

$$
\begin{aligned}
& D_{\mu} \psi_{\nu}^{I}=\left(\partial_{\mu}+\frac{1}{2} \omega_{\mu}^{a} \gamma_{a}\right) \psi_{\nu}^{I}+\partial_{\mu} \phi^{i} Q_{i}^{I J} \psi_{\nu}^{J} \\
& D_{\mu} \chi^{i I}=\left(\partial_{\mu}+\frac{1}{2} \omega_{\mu}^{a} \gamma_{a}\right) \chi^{i I}+\partial_{\mu} \phi^{j}\left(\Gamma_{j k}^{i} \chi^{k I}+Q_{j}^{I J} \chi^{i J}\right) .
\end{aligned}
$$

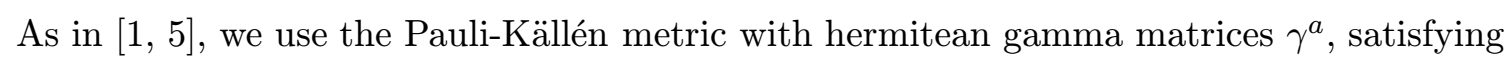
$\gamma_{a} \gamma_{b}=\delta_{a b}+\mathrm{i} \varepsilon_{a b c} \gamma^{c}$. The Lagrangian is invariant under the following supersymmetry transformations

$$
\begin{aligned}
\delta e_{\mu}{ }^{a} & =\frac{1}{2} \bar{\epsilon}^{I} \gamma^{a} \psi_{\mu}^{I}, \\
\delta \psi_{\mu}^{I} & =D_{\mu} \epsilon^{I}-\frac{1}{8} g_{i j} \bar{\chi}^{i I} \gamma^{\nu} \chi^{j J} \gamma_{\mu \nu} \epsilon^{J}-\delta \phi^{i} Q_{i}^{I J} \psi_{\mu}^{J}, \\
\delta \phi^{i} & =\frac{1}{2} \bar{\epsilon}^{I} \chi^{i I}, \\
\delta \chi^{i I} & =\frac{1}{2}\left(\delta^{I J} \mathbf{1}-f^{I J}\right)^{i}{ }_{j} \widehat{\phi} \phi^{j} \epsilon^{J}-\delta \phi^{j}\left(\Gamma_{j k}^{i} \chi^{k I}+Q_{j}^{I J} \chi^{i J}\right),
\end{aligned}
$$

with the supercovariant derivative $\widehat{\partial}_{\mu} \phi^{i} \equiv \partial_{\mu} \phi^{i}-\frac{1}{2} \bar{\psi}_{\mu}^{I} \chi^{i I}$. Observe that the terms proportional to $\delta \phi$ in $\delta \chi^{i I}$ do not satisfy the same constraint (2.10) as $\chi^{i I}$ itself, because the projection operator $\mathbb{P}_{J j}^{I i}$ itself transforms under supersymmetry, such that only the projector condition is supersymmetric.

\section{Isometries and R-symmetries}

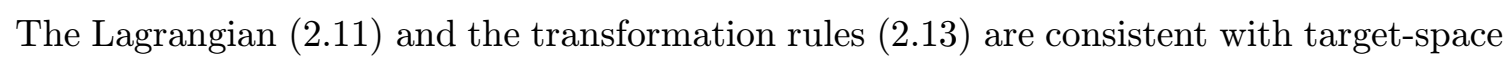
diffeomorphisms and field-dependent $\mathrm{SO}(N)$ R-symmetry rotations. These transformations 
correspond to reparametrizations within certain equivalence classes, but do not, in general, constitute an invariance. The $\mathrm{SO}(N)$ rotations act on $\psi_{\mu}^{I}, \chi^{i I}$ and $Q_{i}^{I J}$ according to

$$
\delta \psi_{\mu}^{I}=\mathcal{S}^{I J}(\phi) \psi_{\mu}^{J}, \quad \delta \chi^{i I}=\mathcal{S}^{I J}(\phi) \chi^{i J}, \quad \delta Q_{i}^{I J}=-D_{i} \mathcal{S}^{I J}(\phi) .
$$

From $(\overline{2} \cdot \overline{2} \cdot \overline{4})$, one concludes that the $f^{I J}$ should be rotated correspondingly,

$$
\delta f^{I J}=2 \mathcal{S}^{K[I}(\phi) f^{J] K} .
$$

The bosonic invariance group $G$ of the Lagrangian (2.11) that commutes with the Lorentz transformations and spacetime diffeomorphisms, is a subgroup of the product of the target-space isometries times the R-symmetry transformations. It is generated by those target-space isometries whose action on the $Q_{i}^{I J}$ and $f^{I J}$ may be absorbed by a special

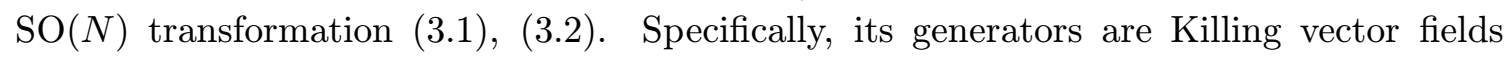
$X^{i}(\phi)$ satisfying

$$
\begin{aligned}
\mathcal{L}_{X} g_{i j}=0, \quad \mathcal{L}_{X} Q_{i}^{I J}+D_{i} \mathcal{S}^{I J}(\phi, X) & =0, \\
\mathcal{L}_{X} f_{i j}^{I J}-2 \mathcal{S}^{K[I}(\phi, X) f_{i j}^{J] K} & =0,
\end{aligned}
$$

where $\mathcal{S}^{I J}(\phi, X)$ is the parameter of an infinitesimal $\mathrm{SO}(N)$ rotation which depends both on $X^{i}(\phi)$ and on the scalar fields. The Lagrangian $\left(\overline{2}_{2}, \overline{1}_{1}\right)$ is then invariant under the combined transformations,

$$
\delta \phi^{i}=X^{i}(\phi), \quad \delta \psi_{\mu}^{I}=\mathcal{S}^{I J}(\phi, X) \psi_{\mu}^{J}, \quad \delta \chi^{i I}=\chi^{j I} \partial_{j} X^{i}+\mathcal{S}^{I J}(\phi, X) \chi^{i J} .
$$

The fermion transformations can be rewritten covariantly,

$$
\begin{aligned}
\delta \psi_{\mu}^{I} & =\mathcal{V}^{I J}(\phi, X) \psi_{\mu}^{J}-\delta \phi^{i} Q_{i}^{I J} \psi_{\mu}^{J}, \\
\delta \chi^{i I} & =D_{j} X^{i} \chi^{j I}+\mathcal{V}^{I J}(\phi, X) \chi^{i J}-\delta \phi^{j}\left(\Gamma_{j k}^{i} \chi^{k I}+Q_{j}^{I J} \chi^{i J}\right),
\end{aligned}
$$

where $\mathcal{V}^{I J}(\phi, X) \equiv X^{j} Q_{j}^{I J}(\phi)+\mathcal{S}^{I J}(\phi, X)$. Using (2.24) and (2.2.3), one verifies that the second equation of (…3.

$$
D_{i} \mathcal{V}^{I J}(\phi, X)=\frac{1}{2} f_{i j}^{I J}(\phi) X^{j}(\phi),
$$

which shows that $\mathcal{V}^{I J}(\phi, X)$ can be regarded as as the moment map associated with the isometry $X^{i}$. After contracting ( $\left(\overline{3} \cdot \bar{b}^{i}\right)$ with $f^{M N i j}$, one obtains

$$
f^{I J i j} D_{i} X_{j}= \begin{cases}\frac{1}{2} d \mathcal{V}^{I J}, & \text { for } N \neq 2,4 \\ \left(d_{+} \mathbb{P}_{+}^{I J, K L}+d_{-} \mathbb{P}_{-}^{I J, K L}\right) \mathcal{V}^{K L}, & \text { for } N=4\end{cases}
$$

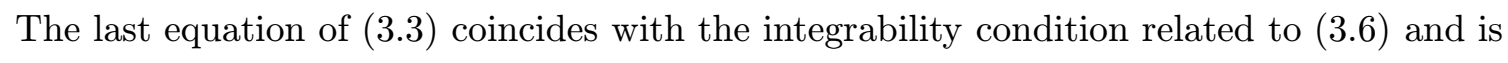
thus automatically satisfied.

For $N>2$, the above analysis shows that there are no obstructions for extending an isometry to an invariance of the Lagrangian. For $N=2$ this is different: $\mathcal{V}^{I J}$ is determined by (13) up to an integration constant related to the invariance of the Lagrangian 
under constant $\mathrm{SO}(2)$ transformations of the fermions. The isometries leave the complex structure invariant and are therefore holomorphic. For $N=4$ the (anti)selfdual almost complex structures $\mathbb{P}_{ \pm}^{I J, K L} f^{K L}$ live in the corresponding $d_{ \pm}$-dimensional quaternion-Kähler subspace. The same holds for the moment maps, $\mathbb{P}_{ \pm}^{I J, K L} \mathcal{V}^{K L}$, which according to $\left(\overline{3} . \overline{3}_{1}^{\prime}\right)$ depend only on the corresponding subspace coordinates. Note, however, that when one of the subspaces is trivial, say when $d_{-}=0$, then $\mathbb{P}_{-}^{I J, K L} \mathcal{V}^{K L}$ corresponds to a triplet of arbitrary constants. This is a consequence of the fact that the model in this case has a rigid $\mathrm{SO}(3)$ invariance acting exclusively on the fermions.

These integration constants in $\mathcal{V}^{I J}$ correspond to the so-called Fayet-Iliopoulos (FI) terms that are known from the gaugings of four-dimensional $N=1$ and $N=2$ supergravity. Indirectly, the above results may have implications for higher-dimensional gauged supergravities, as follows from considering their reduction to three dimensions. For instance, the reduction of $d=4, N=1$ supergravity leads to $d=3, N=2$ supergravity for which the moment maps can always be modified by an additive constant. Consequently, we expect that there are no obstructions against a FI term in four dimensions, which is indeed the case. For $d=4, N=2$ supergravity the situation is more subtle. The reduction of these theories to three dimensions leads to a product of two quaternion-Kähler target spaces, one associated with the vector multiplets and one associated with the hypermultiplets in four dimensions. As in three dimensions there are no integration constants in the moment maps unless one of these quaternion-Kähler spaces is of dimension zero, it follows that FI terms are only possible in four dimensions in the absence of hypermultiplets, a result which is indeed well known.

The generators of $\mathrm{G}$ are labeled by indices $\mathcal{M}, \mathcal{N} \ldots$ and generate an algebra $\mathfrak{g}$. They consist of combined isometries generated by Killing vectors $X^{\mathcal{M} i}$ and infinitesimal $\operatorname{SO}(N)$ rotations $\mathcal{S}^{\mathcal{M} I J} \equiv \mathcal{S}^{I J}\left(\phi, X^{\mathcal{M}}\right)$. For $N=2,4$ one may have the situation that some of the $X^{\mathcal{M}}$ vanish, while the corresponding $\mathcal{S}^{\mathcal{M} I J}$ are constant. Closure of $\mathfrak{g}$ implies,

$$
\begin{aligned}
X^{\mathcal{M} j} \partial_{j} X^{\mathcal{N} i}-X^{\mathcal{N} j} \partial_{j} X^{\mathcal{M} i} & =f^{\mathcal{M N}}{ }_{\mathcal{K}} X^{\mathcal{K} i} \\
{\left[\mathcal{S}^{\mathcal{M}}, \mathcal{S}^{\mathcal{N}}\right]^{I J}-X^{\mathcal{M} i} \partial_{i} \mathcal{S}^{\mathcal{N} I J}+X^{\mathcal{N} i} \partial_{i} \mathcal{S}^{\mathcal{M} I J} } & =-f^{\mathcal{M N}}{ }_{\mathcal{K}} \mathcal{S}^{\mathcal{K} I J}
\end{aligned}
$$

with structure constants $f^{\mathcal{M N}}$.

From the integrability condition of (3.6) one derives that $D_{i} X_{j}-\frac{1}{4} f_{i j}^{M N} \mathcal{V}^{M N}$ commutes with the almost complex structures. For $N>2$ this implies that it can be decomposed in terms of the antisymmetric tensors $h_{i j}^{\alpha}$ introduced in (1,2.8'),

$$
D_{i} X_{j}^{\mathcal{M}}-\frac{1}{4} f_{i j}^{I J} \mathcal{V}^{\mathcal{M} I J} \equiv h_{i j}^{\alpha} \mathcal{V}^{\mathcal{M}}{ }_{\alpha}
$$

Introducing the notation $\mathcal{V}^{\mathcal{M}} i \equiv X^{\mathcal{M} i}$, we establish the following system of linear differential equations,

$$
\begin{aligned}
D_{i} \mathcal{V}^{\mathcal{M} I J} & =\frac{1}{2} f_{i j}^{I J} \mathcal{V}^{\mathcal{M} j} \\
D_{i} \mathcal{V}^{\mathcal{M}}{ }_{\alpha} & =\frac{1}{8} C_{\alpha \beta} h_{i j}^{\beta} \mathcal{V}^{\mathcal{M} j} \\
D_{i} \mathcal{V}^{\mathcal{M}}{ }_{j} & =\frac{1}{4} f_{i j}^{I J} \mathcal{V}^{\mathcal{M} I J}+h_{i j}^{\alpha} \mathcal{V}^{\mathcal{M}}{ }_{\alpha}
\end{aligned}
$$


where the covariant derivative contains the Christoffel connection as well as the $\mathrm{SO}(N) \times \mathrm{H}^{\prime}$ connections. Furthermore, we derive

$$
\begin{aligned}
f^{\mathcal{M N}}{ }_{\mathcal{K}} \mathcal{V}^{\mathcal{K}}{ }^{I J} & =\frac{1}{2} f_{i j}^{I J} \mathcal{V}^{\mathcal{M} i} \mathcal{V}^{\mathcal{N} j}-\left[\mathcal{V}^{\mathcal{M}}, \mathcal{V}^{\mathcal{N}}\right]^{I J} \\
f^{\mathcal{M N}}{ }_{\mathcal{K}} \mathcal{V}^{\mathcal{K}}{ }_{\alpha} & =\frac{1}{8} C_{\alpha \beta} h_{i j}^{\beta} \mathcal{V}^{\mathcal{M} i} \mathcal{V}^{\mathcal{N} j}+f^{\beta \gamma}{ }_{\alpha} \mathcal{V}^{\mathcal{M}}{ }_{\beta} \mathcal{V}^{\mathcal{N}}{ }_{\gamma} \\
f^{\mathcal{M N}}{ }_{\mathcal{K}} \mathcal{V}^{\mathcal{K}}{ }_{i} & =\frac{1}{4} f_{i j}^{I J}\left(\mathcal{V}^{\mathcal{M} I J} \mathcal{V}^{\mathcal{N} j}-\mathcal{V}^{\mathcal{N} I J} \mathcal{V}^{\mathcal{M} j}\right)+h_{i j}^{\alpha}\left(\mathcal{V}^{\mathcal{M}}{ }_{\alpha} \mathcal{V}^{\mathcal{N} j}-\mathcal{V}^{\mathcal{N}}{ }_{\alpha} \mathcal{V}^{\mathcal{M} j}\right)
\end{aligned}
$$

Under the G-transformations the quantities $\mathcal{V}^{\mathcal{M} I J}, \mathcal{V}^{\mathcal{M}}{ }^{i}$ and $\mathcal{V}^{\mathcal{M}}{ }_{\alpha}$ transform according to the adjoint representation of $\mathrm{G}$, up to field-dependent $\mathrm{SO}(N) \times \mathrm{H}^{\prime}$ transformations, as is shown by,

$$
\begin{aligned}
\mathcal{V}^{\mathcal{N} i} D_{i} \mathcal{V}^{\mathcal{M} I J} & =-f^{\mathcal{M N}}{ }_{\mathcal{K}} \mathcal{V}^{\mathcal{K} I J}+\left[\mathcal{V}^{\mathcal{N}}, \mathcal{V}^{\mathcal{M}}\right]^{I J}, \\
\mathcal{V}^{\mathcal{N} i} D_{i} \mathcal{V}^{\mathcal{M}}{ }_{\alpha} & =-f^{\mathcal{M N}}{ }_{\mathcal{K}} \mathcal{V}^{\mathcal{K}}{ }_{\alpha}+f^{\beta \gamma}{ }_{\alpha} \mathcal{V}^{\mathcal{N}}{ }_{\gamma} \mathcal{V}^{\mathcal{M}}{ }_{\beta} .
\end{aligned}
$$

For $\mathcal{V}^{\mathcal{M} i}$ this result is captured by $\left(\overline{3}_{2}-\bar{B}_{i}^{i}\right)$.

\section{Yang-Mills versus Chern-Simons gauged theories}

So far we have been concerned with massless matter fields. We now turn to supersymmetric deformations of these theories that can be obtained by gauging. In that case two issues arise immediately. First the theories discussed so far did not include vector fields that are obviously needed to effect the gauging. Secondly, when the fields are not massless then it is no longer obvious that matter supermultiplets can be exclusively described in terms of scalar and spinor fields, and one might want to include other fields as well. As it turns out, these two issues are somewhat related.

First of all, one can always include vector gauge fields without changing the number of dynamic degrees of freedom, by introducing CS terms. This seems to leave open the option of adding additional standard YM kinetic terms (which may eventually acquire mass terms by spontaneous symmetry breaking) to describe some of the matter degrees of freedom. In fact, all the theories that have been constructed by direct dimensional reduction appear as YM rather than CS gauged theories [3 $3 \overline{3}, 1, \overline{3} \overline{4} \overline{4}]$.

However, it turns out that the YM Lagrangians in three dimensions are simply equivalent to particular CS Lagrangians. The dynamic degrees of freedom are then carried by extra (compensating) scalar fields. In this conversion every gauge field is replaced by two gauge fields and a new scalar field, which together describe the same number of dynamic degrees of freedom as the original gauge field. The nonabelian gauge group is enlarged to a bigger gauge group which is necessarily non-semisimple. To see how this comes about, consider a Lagrangian in three spacetime dimensions with YM kinetic terms quadratic in the field strengths and with moment interactions proportional to gauge covariant tensors $O_{\mu \nu}^{A}$,

$$
\mathcal{L}=-\frac{1}{4} \sqrt{g}\left(F_{\mu \nu}^{A}(A)+O_{\mu \nu}^{A}(A, \Phi)\right) M_{A B}(\Phi)\left(F^{B \mu \nu}(A)+O^{B \mu \nu}(A, \Phi)\right)+\mathcal{L}^{\prime}(A, \Phi) .
$$

Here $A_{\mu}^{A}$ denote the nonabelian gauge fields labeled by indices $A, B, \ldots$, and $\Phi$ generically denotes possible matter fields transforming according to certain representations of the 
gauge group $\mathrm{G}_{\mathrm{YM}}$. The structure constants of this group are denoted by $f_{A B}{ }^{C}$, so that the field strengths read,

$$
F_{\mu \nu}^{A}(A)=\partial_{\mu} A_{\nu}^{A}-\partial_{\nu} A_{\mu}^{A}-f_{B C}{ }^{A} A_{\mu}^{B} A_{\nu}^{C} .
$$

The symmetric matrix $M_{A B}(\Phi)$ may depend on the matter fields and transforms covariantly under $\mathrm{G}_{\mathrm{YM}}$. The last term, $\mathcal{L}^{\prime}(A, \Phi)$, in the Lagrangian is separately gauge invariant and its dependence on the gauge fields is exclusively contained in covariant derivatives of the matter fields or in topological mass terms (i.e. CS terms).

Usually the duality is effected by regarding the field strength as an independent field on which the Bianchi identity is imposed by means of a Lagrange multiplier. Because the Lagrangian (1' proceed differently and write the field strength in terms of new vector fields $B_{A \mu}$ and the derivative of compensating scalar fields $\phi_{A}$, all transforming in the adjoint representation of the gauge group. The explicit expression,

$$
\frac{1}{2} \mathrm{i} \sqrt{g} \varepsilon_{\mu \nu \rho}\left(F^{A \nu \rho}(A)+O^{A \nu \rho}(A, \Phi)\right)=M^{A B}\left(B_{B \mu}-D_{\mu} \phi_{B}\right),
$$

where $M_{A C} M^{C B}=\delta_{A}^{B}$, should be regarded as a field equation that follows from the new

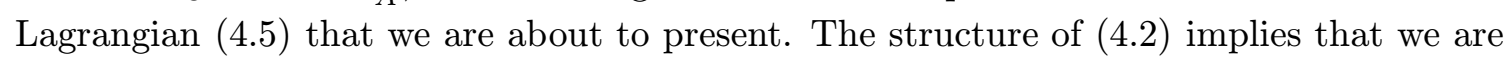
dealing with additional gauge transformations as its right-hand side is invariant under the combined transformations,

$$
\delta B_{A \mu}=D_{\mu} \Lambda_{A}, \quad \delta \phi_{A}=\Lambda_{A},
$$

under which all other fields remain invariant. The corresponding abelian gauge group, $\mathcal{T}$, has nilpotent generators transforming in the adjoint representation of $\mathrm{G}_{\mathrm{YM}}$. Obviously, the $\phi_{A}$ act as compensating fields with respect to $\mathcal{T}$. The combined gauge group is now a semidirect product of $\mathrm{G}_{\mathrm{YM}}$ and $\mathcal{T}$ and its dimension is twice the dimension of the original gauge group $\mathrm{G}_{\mathrm{YM}}$. The covariant field strengths belonging to the new gauge group are $F_{\mu \nu}^{A}(A)$ and $F_{A \mu \nu}(B, A)=2 D_{[\mu} B_{A \nu]}$, and transform under $\mathcal{T}$ according to $\delta F_{\mu \nu}^{A}=0$ and $\delta F_{A \mu \nu}=-\Lambda_{C} f_{A B}{ }^{C} F_{\mu \nu}^{B}$. The fully gauge covariant derivative of $\phi_{A}$ equals

$$
\hat{D}_{\mu} \phi_{A} \equiv D_{\mu} \phi_{A}-B_{A \mu}=\partial_{\mu} \phi_{A}-f_{A B}^{C} A_{\mu}^{B} \phi_{C}-B_{A \mu}
$$

and is invariant under $\mathcal{T}$ transformations.

The field equations corresponding to the new Lagrangian,

$$
\mathcal{L}=-\frac{1}{2} \sqrt{g} \hat{D}_{\mu} \phi_{A} M^{A B}(\Phi) \hat{D}^{\mu} \phi_{B}+\frac{1}{2} \mathrm{i} \varepsilon^{\mu \nu \rho}\left(F_{\mu \nu}^{A} B_{A \rho}-O_{\mu \nu}^{A} \hat{D}_{\rho} \phi_{A}\right)+\mathcal{L}^{\prime}(A, \Phi),
$$

lead to $\left(\overline{1}_{4}^{-} . \overline{2}\right)$ as well as to the same field equations as before for the matter fields $\Phi$. Observe that the Lagrangian is fully gauge invariant up to a total derivative. In this way, the YM Lagrangian has now been converted to a CS Lagrangian, with a different gauge group and a different scalar field content, although the theory is still equivalent on-shell to the original one. To obtain the original Lagrangian (4imply imposes the gauge $\phi_{A}=0$ and integrates out the fields $B_{A \mu}$. 
In figure 1 we schematically illustrate the implications of this equivalence for gauged supergravities in three dimensions. When descended from higher dimensions, the ungauged theories usually appear with the physical bosonic degrees of freedom in different guises, as scalar and vector fields. In order to exhibit possible hidden symmetries, one then dualizes the vector fields after which all bosonic degrees of freedom are represented by scalar fields (in principle there can also exist 'intermediate' versions). The resulting theory is then onshell equivalent to the original one. Both theories can be gauged, but as the table shows, the on-shell equivalence persists.

Perhaps it is worth pointing out that introducing a mass term to a CS theory or a YM

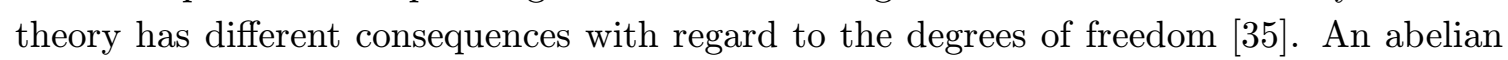
CS term with a regular mass term proportional to $\frac{1}{2} m A_{\mu}^{2}$ yields the following massive wave equation for the vector field,

$$
\partial_{\mu} A_{\nu}-\partial_{\nu} A_{\mu}= \pm \mathrm{i} m \varepsilon_{\mu \nu \rho} A^{\rho}
$$

which describes massive degrees of freedom with spin only equal to +1 or -1 , depending on the sign of the mass term. In contradistinction, a YM kinetic term with a regular mass term $\frac{1}{2} m^{2} A_{\mu}^{2}$ leads to massive degrees of freedom carrying both spin +1 and spin -1 . This doubling of degrees of freedom is consistent with the YM-CS conversion described above, as a YM theory takes the form of a CS theory with twice the number of vector fields.

\section{The embedding tensor}

We now wish to deform the Lagrangian (2.11) such that it becomes invariant under a subset of transformations (1) $(\overline{3})$ with spacetime dependent parameters. The corresponding subalgebra $\mathfrak{g}_{0} \subset \mathfrak{g}$ is characterized by an embedding tensor $\Theta_{\mathcal{M N}}$ via

$$
X^{i}=g \Theta_{\mathcal{M N}} \Lambda^{\mathcal{M}}(x) X^{\mathcal{N} i}, \quad \mathcal{S}^{I J}=g \Theta_{\mathcal{M N}} \Lambda^{\mathcal{M}}(x) \mathcal{S}^{\mathcal{N} I J},
$$

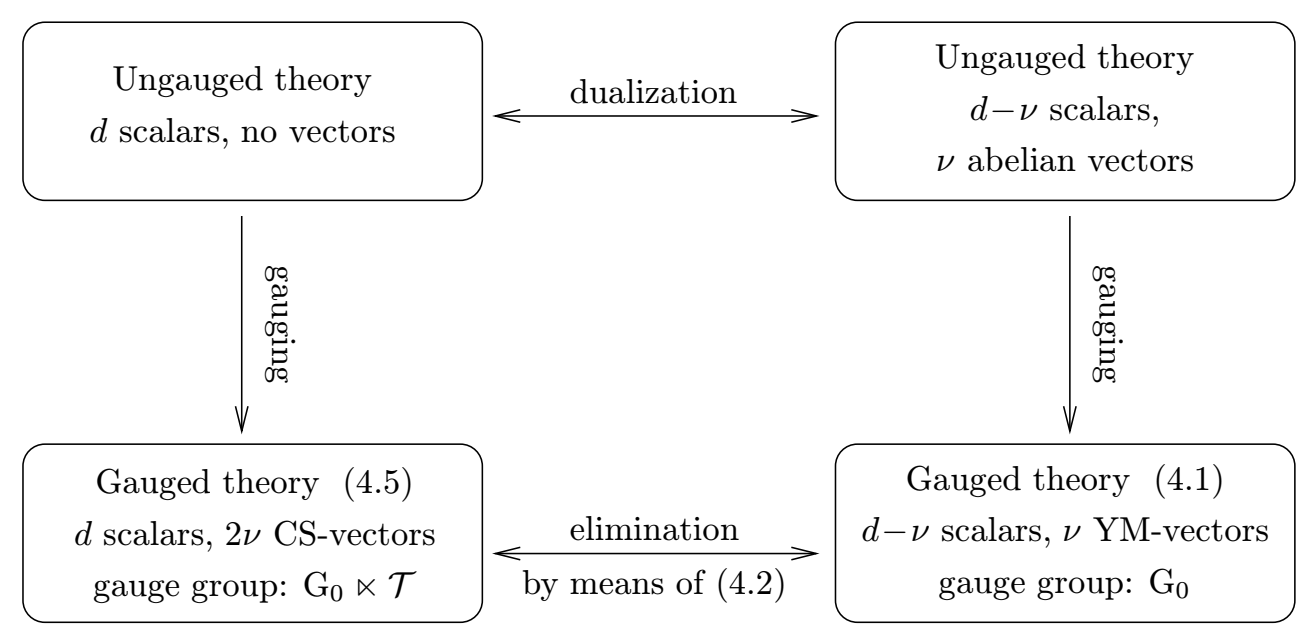

Figure 1: CS and YM gauged supergravity in three dimensions 
with gauge parameters $\Lambda^{\mathcal{N}}(x)$ depending on the spacetime coordinates, and a gauge coupling constant $g$. Unless the gauge group $\mathrm{G}_{0}$ coincides with the full symmetry group $\mathrm{G}$, the embedding tensor acts as a projector which reduces the number of independent parameters according to

$$
\operatorname{dim} \mathfrak{g}_{0}=\operatorname{rank} \Theta .
$$

Although it is not obvious from the way $\Theta_{\mathcal{M N}}$ appears in $(5,1)$ we will see below that it must be gauge invariant and symmetric under interchange of the indices $\mathcal{M}$ and $\mathcal{N}$. Via $t^{\mathcal{M}} \Theta_{\mathcal{M N}} t^{\mathcal{N}}$ it defines an element in the symmetric tensor product $(\mathfrak{g} \otimes \mathfrak{g})_{\text {sym. }}$. In order that the gauge tranformations generate a group, $\Theta_{\mathcal{M N}}$ must satisfy the condition,

$$
\Theta_{\mathcal{M P}} \Theta_{\mathcal{N Q}} f^{\mathcal{P Q}}{ }_{\mathcal{R}}=\hat{f}_{\mathcal{M N}}{ }^{\mathcal{P}} \Theta_{\mathcal{P R}}
$$

for certain constants $\hat{f}_{\mathcal{M N}^{\mathcal{P}}}$, which are subsequently identified as the structure constants of the gauge group. One can verify that the validity of the Jacobi identity for the gauge group structure constants follows directly from the Jacobi identity associated with the group G, subject to projection by the embedding tensor. The symmetry and gauge invariance of $\Theta_{\mathcal{M P}}$ implies that $\hat{f}_{\mathcal{M P}}{ }^{\mathcal{Q}} \Theta_{\mathcal{Q N}}+\hat{f}_{\mathcal{N P}}{ }^{\mathcal{Q}} \Theta_{\mathcal{M Q}}=0$, which can be written in G-covariant form,

$$
\Theta_{\mathcal{P L}}\left(f^{\mathcal{K} \mathcal{L}}{ }_{\mathcal{M}} \Theta_{\mathcal{N} \mathcal{K}}+f^{\mathcal{K} \mathcal{L}}{ }_{\mathcal{N}} \Theta_{\mathcal{M K}}\right)=0 .
$$

Subsequently we introduce the gauge fields $A_{\mu}^{\mathcal{M}}$ into the definition of the covariant derivatives. For example, we have

$$
\mathcal{D}_{\mu} \phi^{i}=\partial_{\mu} \phi^{i}+g \Theta_{\mathcal{M N}} A_{\mu}^{\mathcal{M}} X^{\mathcal{N} i}
$$

for the scalar fields. Their covariant field strengths follow from the commutator of two covariant derivatives, e.g.,

$$
\left[\mathcal{D}_{\mu}, \mathcal{D}_{\nu}\right] \phi^{i}=g \Theta_{\mathcal{M N}} F_{\mu \nu}^{\mathcal{M}} X^{\mathcal{N} i}
$$

and take the form

$$
\Theta_{\mathcal{M N}} F_{\mu \nu}^{\mathcal{M}}=\Theta_{\mathcal{M N}}\left(\partial_{\mu} A_{\nu}^{\mathcal{M}}-\partial_{\nu} A_{\mu}^{\mathcal{M}}-g \hat{f}_{\mathcal{P Q}}{ }^{\mathcal{M}} A_{\mu}^{\mathcal{P}} A_{\nu}^{\mathcal{Q}}\right)
$$

The extra minimal couplings ( tions $(3 . \overline{4}),\left(\begin{array}{l}3 \\ -3\end{array}\right)$ provided we assume the following transformation behavior of the vector fields

$$
\Theta_{\mathcal{M N}} \delta A_{\mu}^{\mathcal{M}}=\Theta_{\mathcal{M N}}\left(-\partial_{\mu} \Lambda^{\mathcal{M}}+g \hat{f}_{\mathcal{P} \mathcal{Q}}^{\mathcal{M}} A_{\mu}^{\mathcal{P}} \Lambda^{\mathcal{Q}}\right)
$$

However, they violate supersymmetry and the central question is whether new terms in the supersymmetry variations and in the Lagrangian can be found such as to regain this symmetry. It is at this point that the need arises to include a CS term for the vector fields,

$$
\mathcal{L}_{\mathrm{CS}}=\frac{1}{4} \mathrm{i} g \varepsilon^{\mu \nu \rho} A_{\mu}^{\mathcal{M}} \Theta_{\mathcal{M N}}\left(\partial_{\nu} A_{\rho}^{\mathcal{N}}-\frac{1}{3} g \hat{f}_{\mathcal{P} \mathcal{Q}}{ }^{\mathcal{N}} A_{\nu}^{\mathcal{P}} A_{\rho}^{\mathcal{Q}}\right),
$$

and assume the following supersymmetry transformations,

$$
\Theta_{\mathcal{M N}} \delta A_{\mu}^{\mathcal{M}}=\Theta_{\mathcal{M N}}\left[2 \mathcal{V}^{\mathcal{M} I J} \bar{\psi}_{\mu}^{I} \epsilon^{J}+\mathcal{V}^{\mathcal{M}}{ }_{i} \bar{\chi}^{i I} \gamma_{\mu} \epsilon^{I}\right]
$$


In order for this to work and to preserve gauge invariance, it is necessary to adopt a symmetric, gauge invariant, embedding tensor.

Although the embedding tensors must be found case by case, let us briefly mention some general properties. For semisimple gaugings, the Lie algebra $\mathfrak{g}_{0}$ always decomposes as a direct sum

$$
\mathfrak{g}_{0}=\bigoplus_{i} \mathfrak{g}_{i} \subset \mathfrak{g},
$$

of simple Lie algebras $\mathfrak{g}_{i}$. In this case, the embedding tensor can be written as a sum of projection operators

$$
\Theta_{\mathcal{M N}}=\sum_{i} \varepsilon_{i} \eta_{\mathcal{M P}}\left(\Pi_{i}\right)^{\mathcal{P}}{ }_{\mathcal{N}}
$$

where $\Pi_{i}$ projects onto the $i$-th simple factor $\mathfrak{g}_{0 i}, \eta_{\mathcal{M P}}$ is the Cartan-Killing form, and the constants $\varepsilon_{i}$ characterize the relative strengths of the gauge couplings. There is only one overall gauge coupling constant $g$ for the maximal theory $(N=16)$, but there may be several independent coupling constants for lower $N$.

For non-semisimple gaugings, $(5,1 \overline{1})$ is replaced by

$$
\mathfrak{g}_{0}=\bigoplus_{i} \mathfrak{g}_{i} \oplus \mathfrak{t}
$$

where $\mathfrak{t}$ represents the solvable part of the gauge group. For the non-semisimple gauge groups which typically appear in theories obtained by dimensional reduction, the latter subalgebra decomposes into

$$
\mathfrak{t}=\mathfrak{t}_{0} \oplus \mathfrak{t}^{\prime}
$$

The abelian subalgebra $\mathfrak{t}_{0}$ here transforms in the adjoint of the semisimple part of the gauge group and pairs up with the semisimple subalgebra in the embedding tensor, which has non-vanishing components only in $\left(\mathfrak{g}_{i} \otimes \mathfrak{t}_{0}\right)_{\text {sym }}$ and in $\left(\mathfrak{t}^{\prime} \otimes \mathfrak{t}^{\prime}\right)_{\text {sym }}$. There are also many examples of nilpotent and almost nilpotent gaugings, where the semisimple part is absent or 'small'. Many examples of non-semisimple gaugings can be generated from semisimple ones by a singular "boost" within the global symmetry group G, as explained in [4īin]

\section{T-tensors, consistency constraints, and the Lagrangian}

Before presenting the full Lagrangian of the gauged supergravity, we define the so-called $T$-tensor (originally introduced in higher-dimensional supergravity [36 $\left.{ }^{3} \bar{i}\right]$ ) as

$$
\begin{aligned}
T^{I J, K L} & \equiv \mathcal{V}^{\mathcal{M} I J} \Theta_{\mathcal{M N}} \mathcal{V}^{\mathcal{N} K L}, & T^{I J i} & \equiv \mathcal{V}^{\mathcal{M} I J} \Theta_{\mathcal{M N}} \mathcal{V}^{\mathcal{N} i}, \\
T^{i j} & \equiv \mathcal{V}^{\mathcal{M} i} \Theta_{\mathcal{M N}} \mathcal{V}^{\mathcal{N} j}, & T_{\alpha}{ }^{i} & \equiv \mathcal{V}^{\mathcal{M}}{ }_{\alpha} \Theta_{\mathcal{M N}} \mathcal{V}^{\mathcal{N} i}, \\
T_{\alpha \beta} & \equiv \mathcal{V}^{\mathcal{M}}{ }_{\alpha} \Theta_{\mathcal{M N}} \mathcal{V}^{\mathcal{N}}{ }_{\beta}, & T^{I J}{ }_{\alpha} & \equiv \mathcal{V}^{\mathcal{M} I J} \Theta_{\mathcal{M N}} \mathcal{V}^{\mathcal{N}}{ }_{\alpha} .
\end{aligned}
$$

The $T$-tensor components that carry indices $\alpha, \beta$ do not appear directly in the Lagrangian and transformation rules and are only defined for $N>2$. From ( $(\overline{5} .4)$ and (3.13) it readily follows that the $T$-tensor transforms covariantly under the gauged isometries. The additional masslike terms and the scalar potential in the Lagrangian and the corresponding 
terms in the supersymmetry variations of the fermion fields, which we will specify shortly, are encoded in three tensors, $A_{1}, A_{2}$ and $A_{3}$, which are related to the $T$-tensor.

A central result of $[\vec{i}] \mid$ is that a gauge group $\mathrm{G}_{0} \subset \mathrm{G}$ with a gauge invariant embedding tensor $\Theta_{\mathcal{M N}}$ describing the minimal couplings according to (").5ist), is consistent with supersymmetry if and only if the associated $T$-tensor $(6.6-1)$ satisfies the constraint,

$$
T^{I J, K L}=T^{[I J, K L]}-\frac{4}{N-2} \delta^{\overparen{[K} T^{L] M, M J}}-\frac{2 \delta^{I[K} \delta^{L] J}}{(N-1)(N-2)} T^{M N, M N} .
$$

For $N=1$ and $N=2$, this constraint degenerates to an identity. The consistency constraint (6) $T^{I J, K L}$ under $\mathrm{SO}(N)$ by

$$
(日 \times 日)_{\mathrm{sym}}=1+\square+\boxminus+\text { 日, }
$$

with each box representing a vector representation of $\mathrm{SO}(N)$, equation $\left(\begin{array}{l}6 \\ 6\end{array} .21\right)$ eliminates the "Weyl-tensor" type representation

$$
\mathbb{P}_{\text {田 }} T^{I J, K L}=0 .
$$

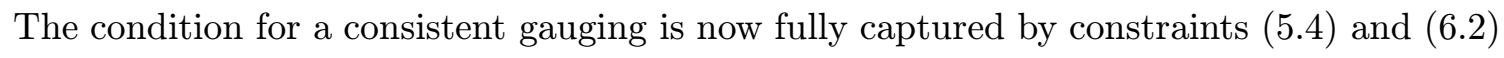
applied to a symmetric embedding tensor $\Theta_{\mathcal{M N}}$. Specific cases will be discussed in later sections.

Let us now present the full Lagrangian and transformation rules. The Lagrangian is given by

$$
\begin{aligned}
\mathcal{L}= & -\frac{1}{2} \mathrm{i} \varepsilon^{\mu \nu \rho}\left(e_{\mu}{ }^{a} R_{\nu \rho a}+\bar{\psi}_{\mu}^{I} \mathcal{D}_{\nu} \psi_{\rho}^{I}\right)-\frac{1}{2} e g_{i j}\left(g^{\mu \nu} \mathcal{D}_{\mu} \phi^{i} \mathcal{D}_{\nu} \phi^{j}+N^{-1} \bar{\chi}^{i I} \not \mathcal{D} \chi^{j I}\right) \\
& +\frac{1}{4} \mathrm{i} g \varepsilon^{\mu \nu \rho} A_{\mu}^{\mathcal{M}} \Theta_{\mathcal{M N}}\left(\partial_{\nu} A_{\rho}^{\mathcal{N}}-\frac{1}{3} g \hat{f}_{\mathcal{P} \mathcal{Q}}{ }^{\mathcal{N}} A_{\nu}^{\mathcal{P}} A_{\rho}^{\mathcal{Q}}\right) \\
& +\frac{1}{4} e g_{i j} \bar{\chi}^{i I} \gamma^{\mu} \gamma^{\nu} \psi_{\mu}^{I}\left(\mathcal{D}_{\nu} \phi^{j}+\widehat{\mathcal{D}}_{\nu} \phi^{j}\right)-\frac{1}{24} e N^{-2} R_{i j k l} \bar{\chi}^{i I} \gamma_{a} \chi^{j I} \bar{\chi}^{k J} \gamma^{a} \chi^{l J} \\
& +\frac{1}{48} e N^{-2}\left(3\left(g_{i j} \bar{\chi}^{i I} \chi^{j I}\right)^{2}-2(N-2)\left(g_{i j} \bar{\chi}^{i I} \gamma^{a} \chi^{j J}\right)^{2}\right) \\
& +e g\left(\frac{1}{2} A_{1}^{I J} \bar{\psi}_{\mu}^{I} \gamma^{\mu \nu} \psi_{\nu}^{J}+A_{2 j}^{I J} \bar{\psi}_{\mu}^{I} \gamma^{\mu} \chi^{j J}+\frac{1}{2} A_{3}^{I J} \bar{\chi}^{i I} \chi^{j J}\right) \\
& -2 e g^{2}\left(g^{i j} A_{2 i}^{I J} A_{2 j}^{I J}-2 N^{-1} A_{1}^{I J} A_{1}^{I J}\right)
\end{aligned}
$$

with covariant derivatives defined by

$$
\begin{aligned}
\mathcal{D}_{\mu} \phi^{i}= & \partial_{\mu} \phi^{i}+g \Theta_{\mathcal{M N}} A_{\mu}^{\mathcal{M}} X^{\mathcal{N} i}, \quad \widehat{\mathcal{D}}_{\mu} \phi^{i}=\mathcal{D}_{\mu} \phi^{i}-\frac{1}{2} \bar{\psi}_{\mu}^{I} \chi^{i I} \\
\mathcal{D}_{\mu} \psi_{\nu}^{I}= & \left(\partial_{\mu}+\frac{1}{2} \omega_{\mu}^{a} \gamma_{a}\right) \psi_{\nu}^{I}+\partial_{\mu} \phi^{i} Q_{i}^{I J} \psi_{\nu}^{J}+g \Theta_{\mathcal{M N}} A_{\mu}^{\mathcal{M}} \mathcal{V}^{\mathcal{N} I J} \psi_{\nu}^{J} \\
\mathcal{D}_{\mu} \chi^{i I}= & \left(\partial_{\mu}+\frac{1}{2} \omega_{\mu}^{a} \gamma_{a}\right) \chi^{i I}+\partial_{\mu} \phi^{j}\left(\Gamma_{j k}^{i} \chi^{k I}+Q_{j}^{I J} \chi^{i J}\right) \\
& +g \Theta_{\mathcal{M N}} A_{\mu}^{\mathcal{M}}\left(\delta_{j}^{i} \mathcal{V}^{\mathcal{N} I J}-\delta^{I J} g^{i k} D_{k} \mathcal{V}^{\mathcal{N}}{ }_{j}\right) \chi^{j J}
\end{aligned}
$$


The supersymmetry transformations read

$$
\begin{aligned}
\delta e_{\mu}{ }^{a} & =\frac{1}{2} \bar{\epsilon}^{I} \gamma^{a} \psi_{\mu}^{I}, \\
\delta A_{\mu}^{\mathcal{M}} & =2 \mathcal{V}^{\mathcal{M} I J} \bar{\psi}_{\mu}^{I} \epsilon^{J}+\mathcal{V}^{\mathcal{M}}{ }_{i} \bar{\chi}^{i I} \gamma_{\mu} \epsilon^{I}, \\
\delta \psi_{\mu}^{I} & =\mathcal{D}_{\mu} \epsilon^{I}-\frac{1}{8} g_{i j} \bar{\chi}^{i I} \gamma^{\nu} \chi^{j J} \gamma_{\mu \nu} \epsilon^{J}-\delta \phi^{i} Q_{i}^{I J} \psi_{\mu}^{J}+g A_{1}^{I J} \gamma_{\mu} \epsilon^{J}, \\
\delta \phi^{i} & =\frac{1}{2} \bar{\epsilon}^{I} \chi^{i I} \\
\delta \chi^{i I} & =\frac{1}{2}\left(\delta^{I J} \mathbf{1}-f^{I J}\right)^{i}{ }_{j} \widehat{\mathcal{D}} \phi^{j} \epsilon^{J}-\delta \phi^{j}\left(\Gamma_{j k}^{i} \chi^{k I}+Q_{j}^{I J} \chi^{i J}\right)-g N A_{2}^{J i I} \epsilon^{J},
\end{aligned}
$$

with

$$
\mathcal{D}_{\mu} \epsilon^{I}=\left(\partial_{\mu}+\frac{1}{2} \omega_{\mu}^{a} \gamma_{a}\right) \epsilon^{I}+\partial_{\mu} \phi^{i} Q_{i}^{I J} \epsilon^{J}+g \Theta_{\mathcal{M N}} A_{\mu}^{\mathcal{M}} \mathcal{V}^{\mathcal{N} I J} \epsilon^{J}
$$

The gauge transformations take the form

$$
\begin{aligned}
\delta \phi^{i} & =g \Theta_{\mathcal{M N}} \Lambda^{\mathcal{M}} X^{\mathcal{N} i} \\
\delta \psi_{\mu}^{I} & =g \Theta_{\mathcal{M N}} \Lambda^{\mathcal{M}} \mathcal{V}^{\mathcal{N} I J} \psi_{\mu}^{J}-\delta \phi^{i} Q_{i}^{I J} \psi_{\mu}^{J} \\
\delta \chi^{i I} & =g \Theta_{\mathcal{M N}} \Lambda^{\mathcal{M}}\left(\chi^{j I} D_{j} \mathcal{V}^{\mathcal{N} i}+\mathcal{V}^{\mathcal{N} I J} \chi^{i J}\right)-\delta \phi^{j}\left(\Gamma_{j k}^{i} \chi^{k I}+Q_{j}^{I J} \chi^{i J}\right) \\
\Theta_{\mathcal{M N}} \delta A_{\mu}^{\mathcal{M}} & =\Theta_{\mathcal{M N}}\left(-\partial_{\mu} \Lambda^{\mathcal{M}}+g \hat{f}_{\mathcal{P Q}}{ }^{\mathcal{M}} A_{\mu}^{\mathcal{P}} \Lambda^{\mathcal{Q}}\right) .
\end{aligned}
$$

For $N>2$, the tensor $A_{1}$ is given by

$$
A_{1}^{I J}=-\frac{4}{N-2} T^{I M, J M}+\frac{2}{(N-1)(N-2)} \delta^{I J} T^{M N, M N} .
$$

In the cases $N=1,2$, this tensor is only partially determined as we shall describe in the next section. For all values of $N$ the tensors $A_{2}$ and $A_{3}$ are given functions of $A_{1}$ and the $T$-tensor $(\underline{6} \cdot \overline{1} \cdot \overline{1})$,

$$
\begin{aligned}
& A_{2 i}^{I J}=\frac{1}{N}\left\{D_{i} A_{1}^{I J}+2 T^{I J}{ }_{i}\right\}, \\
& A_{3 i j}^{I J}=\frac{1}{N^{2}}\left\{-2 D_{(i} D_{j)} A_{1}^{I J}+g_{i j} A_{1}^{I J}+A_{1}^{K[I} f_{i j}^{J] K}\right. \\
& \left.+2 T_{i j} \delta^{I J}-4 D_{[i} T^{I J}{ }_{j]}-2 T_{k[i} f_{j]}^{I J k}\right\} .
\end{aligned}
$$

Using ( implied by their appearance in $(6.6 .5)$ :

$$
A_{1}^{I J}=A_{1}^{J I}, \quad \mathbb{P}_{I i}^{K j} A_{2 j}^{J K}=A_{2 i}^{J I}, \quad A_{3 i j}^{I J}=A_{3 j i}^{J I}=\mathbb{P}_{I i}^{K j} A_{3} \underset{k j}{K J} .
$$

\section{Discussion of low $N$ theories}

In this section we discuss the gauged supergravities for low values of $N$, following [inj]. The cases $N=1,2$ are special because the tensor $A_{1}$ entering the potential and the gravitino mass term is not uniquely determined by the conditions derived in the foregoing section, and 
thus in general is not expressible in terms of the $T$-tensor alone. This leaves the freedom for additional deformations (and thus scalar field potentials) which are not induced by gauging. The additional freedom for $N=1$ and $N=2$ appears via real and complex holomorphic superpotentials, respectively. For $N \geq 3$, on the other hand, all deformations correspond to gaugings.

\section{$7.1 N=1$}

In this case, the target space is a Riemannian manifold of arbitrary dimension $d$. The tensor $A_{1}$ has just one component, which is a gauge invariant function $F(\phi)$ on the target space,

$$
\Theta_{\mathcal{M N}} X^{\mathcal{N} i} \partial_{i} F=0 .
$$

Reading off the values for $A_{2}$ and $A_{3}$ from $(6 . \overline{1} \cdot 1)$, we obtain

$$
A_{1}=F, \quad A_{2 i}=\partial_{i} F, \quad A_{3 i j}=g_{i j} F-2 D_{i} \partial_{j} F+2 T_{i j},
$$

with $T_{i j}=X_{i}^{\mathcal{M}} \Theta_{\mathcal{M N}} X_{j}^{\mathcal{N}}$. As a consequence, any subgroup of isometries can be gauged (for example, by choosing a constant function $F$ ). The gravitino $\psi_{\mu}$ is never charged under the gauge group, and the gauging is restricted to the matter sector. The case $\Theta_{\mathcal{M N}}=0$ and $F \neq 0$ corresponds to deformations of the original theory that are not induced by gaugings. The scalar potential $V$ is given by

$$
V=2 g^{2}\left(g^{i j} \partial_{i} F \partial_{j} F-2 F^{2}\right)
$$

so that the function $F$ serves as the real superpotential. Stationary points of $F$ define (anti-de Sitter) supersymmetric ground states.

\section{$7.2 N=2$}

The target space in this case is a Kähler manifold and may be conveniently parametrized by $d / 2$ complex coordinates and their conjugates, $\left(\phi^{i}, \bar{\phi}^{\bar{\imath}}\right)$. Its metric and the $\mathrm{SO}(2)$ connection are given in terms of the Kähler $K(\phi, \bar{\phi})$ potential as $g_{i \bar{\jmath}}=\partial_{i} \partial_{\bar{\jmath}} K, Q_{i} \equiv Q_{i}^{12}=-\frac{1}{4} \mathrm{i} \partial_{i} K$. Any subgroup of the invariance group can be gauged. Partial results for abelian $N=2$

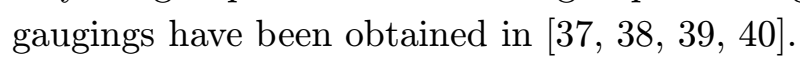

According to (3) only holomorphic isometries of the target space can be extended to symmetries of the Lagrangian. Such isometries are parameterized by holomorphic Killing vector fields $\left(X^{i}, X^{\bar{\imath}}\right)$,

$$
\partial_{\bar{\imath}} X^{j}=0, \quad D_{i} X_{\bar{\jmath}}+D_{\bar{\jmath}} X_{i}=0
$$

The second condition implies that the Kähler potential remains invariant under the isometry up to a Kähler transformation. We write this special Kähler transformation in terms of a holomorphic function $\mathcal{S}(\phi)$, i.e.,

$$
\delta K(\phi, \bar{\phi})=-X^{i} \partial_{i} K-X^{\bar{\imath}} \partial_{\bar{\imath}} K=4 \mathrm{i}(\mathcal{S}-\overline{\mathcal{S}})
$$


Equation (3.6ij) may then be solved as

$$
\mathcal{V} \equiv \mathcal{V}^{12}=-\frac{1}{4} \mathrm{i}\left(X^{i} \partial_{i} K-X^{\bar{\imath}} \partial_{\bar{\imath}} K\right)+\mathcal{S}^{12}=-\frac{1}{2} \mathrm{i} X^{i} \partial_{i} K+2 \mathcal{S}
$$

For every generator $X^{\mathcal{M}}$ of the invariance group we thus identify a holomorphic function $\mathcal{S}^{\mathcal{M}}$, determined by $\left(\overline{\bar{T}_{1}} . \overline{4}_{1}\right)$ up to a real constant. The particular transformation (which we denote with the extra label $\mathcal{M}=0$ ),

$$
X^{0 i}=0, \quad \mathcal{S}^{0}=\frac{1}{2}, \quad \mathcal{V}^{0}=1,
$$

constitutes a central extension of the isometry group and generates the $\mathrm{SO}(2) \mathrm{R}$-symmetry group that acts exclusively on the fermions. These symmetries play a role in the presence of FI terms. We refer to [i.j] for further details.

For the $T$-tensor, we introduce the notation

$$
T \equiv T^{I J, I J}=2 T^{12,12}, \quad T_{i} \equiv T_{i}^{12}=\frac{1}{2} \mathrm{i} \partial_{i} T .
$$

The tensor $A_{1}^{I J}$ is determined by $(6.10)$

$$
A_{1}^{11}=-T-e^{K / 2} \Re W, \quad A_{1}^{22}=-T+e^{K / 2} \Re W, \quad A_{1}^{12}=A_{1}^{21}=e^{K / 2} \Im W,
$$

with an holomorphic superpotential $W(\phi)$, which, because of gauge covariance, must satisfy

$$
\Theta_{\mathcal{M N}}\left(X^{\mathcal{N} i} D_{i} W-2 \mathrm{i} \mathcal{V}^{\mathcal{N}} W\right)=\Theta_{\mathcal{M N}}\left(X^{\mathcal{N} i} \partial_{i} W-4 \mathrm{i} \mathcal{S}^{\mathcal{N}} W\right)=0,
$$

with the Kähler covariant derivative $D_{i} W \equiv \partial_{i} W+\partial_{i} K W$. The tensors $A_{2}, A_{3}$ follow from (6.1 61 ); for $A_{2}$ we find,

$$
A_{2 i}^{11}=-\mathrm{i} A_{2 i}^{12}=-\frac{1}{2}\left(\partial_{i} T+e^{K / 2} D_{i} W\right), \quad A_{2 i}^{21}=\mathrm{i} A_{2 i}^{22}=\frac{1}{2} \mathrm{i}\left(\partial_{i} T-e^{K / 2} D_{i} W\right) .
$$

The scalar potential of the gauged theory is given by

$$
V=g^{2}\left(4 g^{i \bar{\imath}} \partial_{i} T \partial_{\bar{\imath}} T-4 T^{2}+g^{i \bar{\imath}} e^{K} D_{i} W D_{\bar{\imath}} \bar{W}-4 e^{K}|W|^{2}\right) .
$$

Note that in three dimensions, the scalar potential contains terms quartic in the moment map $\mathcal{V}$, since the $T$-tensor is quadratic in $\mathcal{V}$. This is in contrast with e.g. four dimensions, where the corresponding part of the scalar potential is quadratic in $\mathcal{V}$.

Analogous to the $N=1$ case, there are two kinds of supersymmetric deformations of the original theory. On the one hand, there are the gaugings, which are completely characterized by an embedding tensor $\Theta_{\mathcal{M N}}$. The above analysis shows that there is no restriction on the $T$-tensor, and therefore any subgroup of the invariance group of the theory is an admissible gauge group, as long as its embedding tensor satisfies (15.). On the other hand there are the deformations described by the holomorphic superpotential $W$, which are not induced by a gauging. In case both deformations are simultaneously present, their compatibility requires ( $(\overline{7} . \overline{8})$. Pure $N=2$ supergravity (without gauging) can have a cosmological constant corresponding to a constant $W$ and vanishing $T$. This implies that the gravitino mass matrix is traceless. An alternative way to generate a cosmological term in pure supergravity makes use of gauging the R-symmetry group. In that case, $T$ equals a nonzero constant (equal to $\Theta_{00}$ ) and $W=0$; the gravitino mass matrix is then proportional to the identity. The latter version has been considered in [3근] 


\subsection{Some comments on $N=3$ and $N=4$ theories}

For $N=3$, the target space is a quaternion-Kähler manifold. In this case, the consistency condition ( consistently gauged. For $N=4$ on the other hand, the target space is locally a product of two quaternion-Kähler manifolds of dimension $d_{ \pm}$. The almost-complex structures $f^{I J}$ decompose into two sets of three almost-complex structures $f^{ \pm}$,

$$
\begin{aligned}
& f^{+P} \equiv \frac{1}{2}(J+1) f^{P}=\frac{1}{2} f^{P}-\frac{1}{4} \epsilon^{P Q R} f^{Q R}, \quad(P=1,2,3), \\
& f^{-\bar{P}} \equiv \frac{1}{2}(J-1) f^{P}=-\frac{1}{2} f^{P}-\frac{1}{4} \epsilon^{P Q R} f^{Q R}, \quad(\bar{P}=1,2,3),
\end{aligned}
$$

corresponding to the decomposition of the $\mathrm{SO}(4)$ R-symmetry group,

$$
\mathrm{SO}(4)=\mathrm{SO}(3)^{+} \times \mathrm{SO}(3)^{-} \text {. }
$$

In this basis, the consistency condition $\left(\overline{6}, \overline{2}{ }^{2}\right)$ that encodes supersymmetry of the theory takes the form

$$
T^{P Q}=\frac{1}{3} \delta^{P Q} T^{R R}, \quad \text { where } \quad T^{P Q}=\mathcal{V}^{\mathcal{M} P} \Theta_{\mathcal{M N}} \mathcal{V}^{\mathcal{N} Q}
$$

and correspondingly for $T^{\bar{P} \bar{Q}}$. The off-diagonal components, $T^{P \bar{Q}}$ are constrained by the

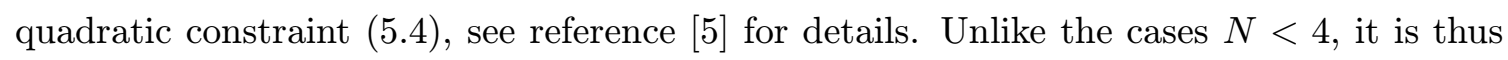
no longer possible to gauge any subgroup of the isometry group. We refer to $[\bar{i} \mid \overline{0}]$ for further details. Henceforth, we will call a subgroup of $G$ 'admissible' if its embedding tensor obeys $\left(\overline{5}-\overline{5}_{3}\right)$ and $\left(\overline{6} . \overline{2}^{\prime}\right)$, so that supersymmetry is preserved.

\section{Symmetric target spaces with $N>4$}

Beyond $N=4$, the only admissible target spaces are the symmetric spaces listed in table Hence they are coset spaces $\mathrm{G} / \mathrm{H}$, where the isotropy group is equal to the (maximal) holonomy group $\mathrm{SO}(N) \times \mathrm{H}^{\prime}$. The scalar fields may be described by means of a G-valued matrix $L\left(\phi^{i}\right)$, on which the rigid action of $\mathrm{G}$ is realized by left multiplication, while $\mathrm{SO}(N) \times$ $\mathrm{H}^{\prime}$ acts as a local symmetry by multiplication from the right. The generators of the group $\mathrm{G}$ constitute a Lie algebra $\mathfrak{g}$, which thus decomposes into $\left\{t^{\mathcal{M}}\right\}=\left\{X^{I J}, X^{\alpha}, Y^{A}\right\}$. The $X^{I J}$ generate $\mathrm{SO}(N)$, the $X^{\alpha}$ generate the compact group $\mathrm{H}^{\prime}$, while the remaining (noncompact) generators $Y^{A}$ transform in a spinor representation of $\mathrm{SO}(N)$. The connection with the general quantities introduced above is given via

$$
\begin{aligned}
L^{-1} \partial_{i} L & =\frac{1}{2} Q_{i}^{I J} X^{I J}+Q_{i}^{\alpha} X^{\alpha}+e_{i}{ }^{A} Y^{A}, \\
X^{\mathcal{M} i} \partial_{i} L & =t^{\mathcal{M}} L-\frac{1}{2} \mathcal{S}^{\mathcal{M} I} L X^{I J}+\mathcal{S}^{\mathcal{M} \alpha} L X^{\alpha}, \\
L^{-1} t^{\mathcal{M}} L & =\frac{1}{2} \mathcal{V}^{\mathcal{M} I J} X^{I J}+\mathcal{V}^{\mathcal{M}}{ }_{\alpha} X^{\alpha}+\mathcal{V}^{\mathcal{M}}{ }_{A} Y^{A}, \\
g_{i j} & =e_{i}{ }^{A} e_{j}{ }^{B} \delta_{A B}, \quad f_{i j}^{I J}=-\Gamma_{A B}^{I J} e_{i}^{A} e_{j}^{B}, \quad \mathcal{V}^{\mathcal{M}}{ }_{i}=e_{i}{ }^{A} \mathcal{V}^{\mathcal{M}}{ }_{A} .
\end{aligned}
$$

Equations (3.12) then correspond to the fact that the map

$$
t^{\mathcal{M}} \rightarrow L^{-1} t^{\mathcal{M}} L
$$


is an isomorphism of the algebra $\mathfrak{g}$; the actual equations follow straightforwardly from the commutator $\left[L^{-1} t^{\mathcal{M}} L, L^{-1} t^{\mathcal{N}} L\right]$, upon using the explicit commutation relations of the generators $X^{I J}, X^{\alpha}$ and $Y^{A}$. Linear first-order differential equations such as (3.11) can be

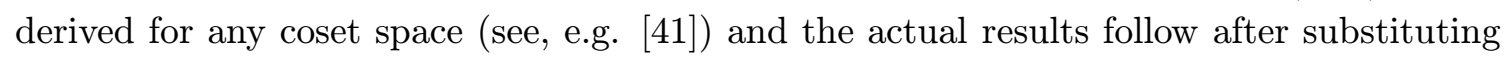
the appropriate expressions for the coset-space curvatures. Here we should add that the above analysis can be straightforwardly extended to $N=4$ with symmetric target spaces, as all these spaces are known and exhibit the same characteristics as outlined above.

The symmetric space structure in particular implies, that the $T$-tensor $\left(\overline{6}_{0} \bar{x}_{i}^{\prime}\right)$ coincides with the image of the embedding tensor $\Theta_{\mathcal{M N}}$ under $(\overline{8}, \overline{2} . \overline{2} i)$,

$$
T_{\mathcal{A B}}=\mathcal{V}^{\mathcal{M}}{ }_{\mathcal{A}} \Theta_{\mathcal{M N}} \mathcal{V}^{\mathcal{N}}{ }_{\mathcal{B}}
$$

This allows us to lift the consistency condition (6. resentation $\boxplus$ in the $T$-tensor vanishes, to a field-independent condition on the embedding tensor,

$$
\mathbb{P}_{\mathcal{M N}}^{\mathcal{P Q}} \Theta_{\mathcal{P Q}}=0
$$

Here $\mathbb{P}$ projects onto the unique irreducible representation in $(\mathfrak{g} \otimes \mathfrak{g})_{\text {sym }}$ that contains the $\boxplus$ representation of the $T$-tensor, via $\left(\overline{8}^{-} \cdot \overline{3}\right)$. It is a non-trivial fact that the $T$-tensor, which is assigned to R-symmetry representations, and appears in the fermionic masslike terms and the scalar potential, can be assembled into representations of the global symmetry group $\mathrm{G}$, as was first noticed in the context of maximal gauged supergravity in four dimensions [i] ${ }^{1} \overline{6}_{1}^{\prime}$. For the symmetric target spaces, admissible subgroups of $\mathrm{G}$ are characterized by an embedding tensor that obeys $\left({ }_{5}^{5} .3\right)$ and $(\overline{8} \cdot 4)$.

Note that the consistency conditions (15. plexified global symmetry group $\mathrm{G}_{\mathbb{C}}$. Indeed, non-semisimple gaugings in four dimensions

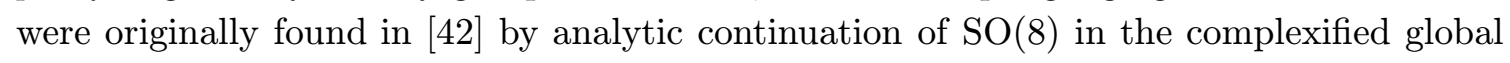
symmetry group $\mathrm{E}_{7}(\mathbb{C})$. In three dimensions, a similar construction should exist relating the different non-compact real forms of the gauge groups listed in table $\underline{2}$ i below, and explaining why ratios of coupling constants between the factor groups remain the same.

\section{Admissible gauge groups for $N=16$}

To illustrate the variety of possible gaugings, we now turn to the maximally extended $N=16$ supergravity. In this case the embedding tensor transforms as an element of the symmetric tensor product of two adjoint (and in this case also fundamental) representations of $\mathrm{E}_{8(8)}$

$$
(248 \otimes \mathbf{2 4 8})_{\mathrm{sym}}=\mathbf{1} \oplus \mathbf{3 8 7 5} \oplus \mathbf{2 7 0 0 0},
$$

As shown in [2]

$$
\left(\mathbb{P}_{\mathbf{2 7 0 0 0}}\right)_{\mathcal{M N}}^{\mathcal{P Q}} \Theta_{\mathcal{P Q}}=0 .
$$

so that the embedding tensor decomposes into a singlet and the $\mathbf{3 8 7 5}$ representations of $\mathrm{E}_{8(8)}$. Following $[\underline{\underline{g}}]$, we split the generators of $\mathfrak{g}=\mathfrak{e}_{8(8)}$ into 120 compact ones $X^{I J}=-X^{J I}$ 
with $\mathrm{SO}(16)$ vector indices $I, J=1, \ldots, 16$, and 128 noncompact ones $\left\{Y^{A}\right\}$ with $\mathrm{SO}(16)$ spinor indices $A=1, \ldots 128$. Then the condition $\left(\overline{9}, \overline{2} 2_{1}^{\prime}\right)$ implies that only special $\mathrm{SO}(16)$ representations can appear in $\Theta$; we have

$$
\Theta=\Theta_{I J \mid K L} X^{I J} \otimes X^{K L}+\Theta_{I J \mid A}\left(X^{I J} \otimes Y^{A}+Y^{A} \otimes X^{I J}\right)+\Theta_{A \mid B} Y^{A} \otimes Y^{B},
$$

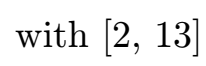

$$
\begin{aligned}
\Theta_{I J \mid K L} & =-2 \theta \delta_{K L}^{I J}+2 \delta_{I[K} \Xi_{L] J}+\Xi_{I J K L}, \\
\Theta_{I J \mid A} & =-\frac{1}{7} \Gamma_{A \dot{A}}^{[I} \Xi^{J] \dot{A}}, \\
\Theta_{A \mid B} & =\theta \delta_{A B}+\frac{1}{96} \Xi_{I J K L} \Gamma_{A B}^{I J K L},
\end{aligned}
$$

and the $\mathrm{SO}(16) \Gamma$ matrices $\Gamma_{A \dot{A}}^{I}$, where the indices $\dot{A}=1, \ldots, 128$ label the conjugate spinor representation. The tensors $\Xi_{I J}, \Xi_{I J K L}$ and $\Xi^{I \dot{A}}$ transform as the 135, 1820 and $\overline{\mathbf{1 9 2 0}}$ representations of $\mathrm{SO}(16)$, respectively; hence $\Xi_{I I}=0=\Gamma_{A \dot{A}}^{I} \Xi^{I \dot{A}}$, and $\Xi_{I J K L}$ is completely antisymmetric in its four indices. The singlet contribution in $(\overline{\overline{9}} . \overline{4} \overline{4})$ is absent for non-semisimple and complex gauge groups.

Although the solutions to (2.1) have not been exhaustively classified, it is known that all the irreducible components occurring in (19.4) can and do appear, depending on the type of gauge group. The simplest examples are the semisimple gaugings with maximal supersymmetry constructed in $[\overline{2}]$, for which we have quite generally

$$
\left.\theta, \Xi_{I J}, \Xi_{I J K L} \neq 0 \text { and } \Xi^{I \dot{A}}=0 \quad \text { (for semisimple } \mathfrak{g}_{0}\right) .
$$

In this case, the sum (5.12i) contains at most two terms, i.e. the gauge groups are typically products of two simple groups $\mathrm{G}_{1} \times \mathrm{G}_{2}$ with a fixed ratio of coupling constants $g_{1} / g_{2}$, such that there is only one free parameter in the theory. Schematically, we have the admissible gauge groups

$$
G_{0}=E_{8}, E_{7} \times A_{1}, E_{6} \times A_{2}, F_{4} \times G_{2}, D_{4} \times D_{4} .
$$

which appear in all those real forms that are consistent with $E_{8(8)}$. Remarkably, the ratio $g_{1} / g_{2}$ does not depend on the chosen real form. Furthermore, as shown in [2: all these theories possess maximally supersymmtric (AdS or Minkowski) ground states. The corresponding theories with their corresponding gauge groups, which are particular

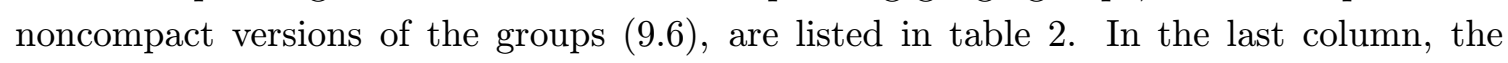
table lists the symmetry groups of the ground states, which are superextensions of the three-dimensional AdS group $\mathrm{SL}(2, \mathbb{R}) \times \mathrm{SL}(2, \mathbb{R})$. Besides the fully supersymmetric vacua, there are also many known stationary points with partially broken supersymmetry $\left[1 \overline{2} \overline{2}_{-1}^{\prime}\right.$

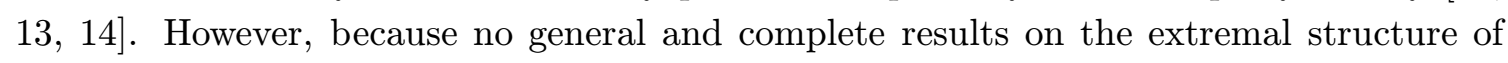
the associated potentials are available to date ${ }^{2}$, many further extremal points could exist besides the known ones.

\footnotetext{
${ }^{2}$ Even for $D=4$, the complexity of the potentials has prevented the identification of new stationary points beyond those already found in [15, i1 in, although the potentials are now known on a larger manifold of scalar field configurations thanks to the high performance symbolic algebra program developed in [i]
} 


\begin{tabular}{||c|c|c|c||}
\hline gauge group $\mathrm{G}_{0}$ & ratio $g_{1} / g_{2}$ & $\left(n_{\mathrm{L}}, n_{\mathrm{R}}\right)$ & ground state symmetry group \\
\hline \hline $\mathrm{SO}(8) \times \mathrm{SO}(8)$ & $g_{1} / g_{2}=-1$ & $(8,8)$ & $\mathrm{OSp}(8 \mid 2, \mathbb{R}) \times \mathrm{OSp}(8 \mid 2, \mathbb{R})$ \\
\hline $\mathrm{SO}(7,1) \times \mathrm{SO}(7,1)$ & $g_{1} / g_{2}=-1$ & $(8,8)$ & $\mathrm{F}(4) \times \mathrm{F}(4)$ \\
\hline $\mathrm{SO}(6,2) \times \mathrm{SO}(6,2)$ & $g_{1} / g_{2}=-1$ & $(8,8)$ & $\mathrm{SU}(4 \mid 1,1) \times \mathrm{SU}(4 \mid 1,1)$ \\
\hline $\mathrm{SO}(5,3) \times \mathrm{SO}(5,3)$ & $g_{1} / g_{2}=-1$ & $(8,8)$ & $\mathrm{OSp}\left(4^{*} \mid 4\right) \times \mathrm{OSp}\left(4^{*} \mid 4\right)$ \\
\hline $\mathrm{SO}(4,4) \times \mathrm{SO}(4,4)$ & $g_{1} / g_{2}=-1$ & $(8,8)$ & $\operatorname{Minkowski} \operatorname{vacuum}$ \\
\hline $\mathrm{G}_{2(2)} \times \mathrm{F}_{4(4)}$ & $g_{\mathrm{G}_{2}} / g_{\mathrm{F}_{4}}=-3 / 2$ & $(4,12)$ & $\mathrm{D}^{1}\left(2,1 ;-\frac{2}{3}\right) \times \mathrm{OSp}\left(4^{*} \mid 6\right)$ \\
\hline $\mathrm{G}_{2} \times \mathrm{F}_{4(-20)}$ & $g_{\mathrm{G}_{2}} / g_{\mathrm{F}_{4}}=-3 / 2$ & $(7,9)$ & $\mathrm{G}(3) \times \mathrm{OSp}(9 \mid 2, \mathbb{R})$ \\
\hline $\mathrm{E}_{6(6)} \times \mathrm{SL}(3)$ & $g_{\mathrm{A}_{2}} / g_{\mathrm{E}_{6}}=-2$ & $(16,0)$ & $\mathrm{OSp}\left(4^{*} \mid 8\right) \times \mathrm{SU}(1,1)$ \\
\hline $\mathrm{E}_{6(2)} \times \mathrm{SU}(2,1)$ & $g_{\mathrm{A}_{2}} / g_{\mathrm{E}_{6}}=-2$ & $(12,4)$ & $\mathrm{SU}(6 \mid 1,1) \times \mathrm{D}^{1}\left(2,1 ;-\frac{1}{2}\right)$ \\
\hline $\mathrm{E}_{6(-14)} \times \mathrm{SU}(3)$ & $g_{\mathrm{A}_{2}} / g_{\mathrm{E}_{6}}=-2$ & $(10,6)$ & $\mathrm{OSp}(10 \mid 2, \mathbb{R}) \times \mathrm{SU}(3 \mid 1,1)$ \\
\hline $\mathrm{E}_{7(7)} \times \mathrm{SL}(2)$ & $g_{\mathrm{A}_{1}} / g_{\mathrm{E}_{7}}=-3$ & $(16,0)$ & $\mathrm{SU}(8 \mid 1,1) \times \mathrm{SU}(1,1)$ \\
\hline $\mathrm{E}_{7(-5)} \times \mathrm{SU}(2)$ & $g_{\mathrm{A}_{1}} / g_{\mathrm{E}_{7}}=-3$ & $(12,4)$ & $\mathrm{OSp}(12 \mid 2, \mathbb{R}) \times \mathrm{D}^{1}\left(2,1 ;-\frac{1}{3}\right)$ \\
\hline $\mathrm{E}$ & $g_{8(8)}$ & $(16,0)$ & $\mathrm{OSp}(16 \mid 2, \mathbb{R}) \times \mathrm{SU}(1,1)$ \\
\hline $\mathrm{E}_{8}$ & &
\end{tabular}

Table 2: The $N=16$ theories with semisimple gauge groups $G_{0}$. Except for the last row, the gauge groups appear as direct products of two factors whose coupling constant ratio $g_{1} / g_{2}$ is determined by $(9.2)$. All these theories admit a maximally supersymmetric AdS (or Minkowski, for $\mathrm{G}_{0}=$ $\mathrm{SO}(4,4) \times \mathrm{SO}(4,4))$ ground state, whose symmetry group factorizes according to $\mathrm{G}_{\mathrm{L}} \times \mathrm{G}_{\mathrm{R}}$, as specified in the last column; the supercharges split accordingly into $n_{\mathrm{L}}+n_{\mathrm{R}}=16$.

A second class are the non-semisimple gaugings, whose existence can also be inferred from the fact that in higher dimensions there are many maximal gaugings with non-

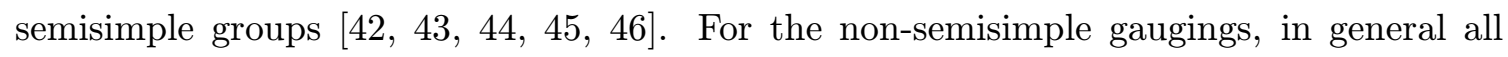
components of the embedding tensor in (19.9) are non-vanishing, in particular the 'offdiagonal' components (mixing compact and non-compact generators)

$$
\Xi^{I \dot{A}} \neq 0 \quad\left(\text { for non-semisimple } \mathfrak{g}_{0}\right) \text {. }
$$

For $N=16$, the most prominent examples are [i]

$$
\begin{aligned}
& \mathrm{G}_{0}=\mathrm{SO}(p, q) \ltimes \mathrm{T}_{28} \quad \text { for } p+q=8 ; \\
& \mathrm{G}_{0}=\mathrm{CSO}(p, q ; r) \ltimes \mathrm{T}_{p, q, r} \quad \text { for } p+q+r=8 \text { and } r>0
\end{aligned}
$$

Here, $\mathrm{T}_{28}$ is an abelian group of 28 translations transforming in the adjoint of $\mathrm{SO}(p, q)$. Similarly, $\mathrm{T}_{p, q, r}$ is a group of translations, but of smaller dimension

$$
\operatorname{dim} \mathrm{T}_{p, q, r}=\operatorname{dim} \operatorname{CSO}(p, q ; r)=28-\frac{1}{2} r(r-1) .
$$


Note that the groups in $(\overline{9} . \overline{8})$ involving $\mathrm{SO}(p, q)$ or $\mathrm{CSO}(p, q ; r)$ with $p \neq 0,8$ admit only one embedding, whereas there are two inequivalent $S O(8) \ltimes T_{28}$ gaugings, corresponding to the compactifications IIA and IIB supergravity on $S^{7}$. Quite generally, reduction of a higher-dimensional gauged supergravity (with semisimple or non-semisimple gauge group) on a torus will always lead to a non-semisimple gauge group in three dimensions. In view of the equivalence of CS and YM type gauge theories explained in section 4, the gauged supergravities with the gauge groups $\left(\overline{9}-\bar{g}^{\prime}\right)$ are consequently on-shell equivalent to the ones obtained by reducing the $S O(p, q)$ and $C S O(p, q ; r)$ theories of [i풀] of non-semisimple gaugings can be generated from semisimple ones by the boost method described in [4]

In contrast to the semisimple gaugings, the non-semisimple ones do not admit maximally supersymmetric groundstates. The potentials contain exponential factors and their minimum is usually reached at infinity. This phenomenon is well-known from higherdimensional gauged supergravities. The non-existence of fully supersymmetric vacua is also related to the disappearance of the supersymmetric vacuum that is known to occur when one reduces maximal gauged supergravity from four or five to three dimensions on a torus.

The most curious solution of the consistency conditions is the complex gauge group

$$
\mathrm{G}_{0}=\mathrm{SO}(8, \mathbb{C})
$$

which can be realized in two inequivalent ways, corresponding to two possible and inequivalent embeddings of $\mathrm{SO}\left(8, \mathbb{C}\right.$ ) into the (real) Lie group $\mathrm{E}_{8(8)}$ (there are similar complex gauge groups $\mathrm{SO}(n, \mathbb{C})$ for $N=2 n=12,10$ supergravities). This gauging provides an example of a purely off-diagonal embedding tensor for which

$$
\theta=\Xi_{I J}=\Xi_{I J K L}=0 \quad \text { and } \quad \Xi^{I \dot{A}} \neq 0 \quad\left(\text { for } \mathfrak{g}_{0}=\mathfrak{s o}(8, \mathbb{C})\right),
$$

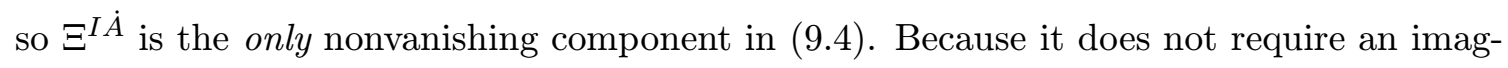
inary unit, this embedding exhibits some rather strange properties. Like the semisimple gauge groups of table $\underline{\overline{2}}$, the $\mathrm{SO}(8, \mathbb{C})$ gauged supergravities cannot be derived from higher dimensions by any known mechanism. Furthermore, they feature a de Sitter stationary point at the origin breaking all supersymmetries, and with tachyonic instabilities. (There are indications that these models possess no further extrema besides the one at the origin. ${ }^{3}$ ) We note that CS gauge theories with complex gauge groups are of considerable

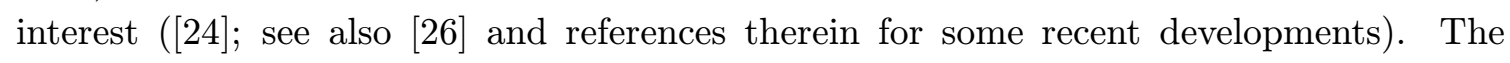
embedding of such theories into supergravity with non-trivial matter couplings may well provide interesting new perspectives.

As we already explained in the introduction, the existence of the large variety of gauged supergravities in three space-time dimensions, with potentials that have stationary points corresponding to AdS backgrounds, is important in the context of the AdS/CFT correspondence. In the case at hand the correspondence implies a relation between an AdS solution of a certain three-dimensional gauged supergravity and a two-dimensional

\footnotetext{
${ }^{3}$ T. Fischbacher, private communication.
} 
(super)conformal theory living on the boundary of the AdS space. The two-dimensional theories are characterized by an infinite-dimensional superconformal algebra. These algebras have all been classified [i] $\left.\bar{z}_{-}\right]$; they consist of a sum of two algebras, pertaining to the left- and right-moving sectors, respectively, containing an $n_{\mathrm{L}^{-}}$and an $n_{\mathrm{R}^{-}}$superextended Virasoro algebra. On the supergravity side, the maximal finite-dimensional subalgebra will correspond to the symmetry algebra of the $\mathrm{AdS}_{3}$ stationary point. To illustrate this, one may consider the theories listed in table $\underline{2}$, , which admit maximally supersymmetric $\mathrm{AdS}_{3}$ stationary points whose symmetry algebra are listed in the last column. Indeed, each of these symmetry algebras coincides with the maximal finite subalgebra of a corresponding superconformal algebra of [i $\left.\bar{i}_{\overline{1}}\right]$ with the appropriate numbers, $n_{\mathrm{L}}$ and $n_{\mathrm{R}}$, of supercharges.

The infinite-dimensional superconformal algebras appear in the asymptotic symmetries

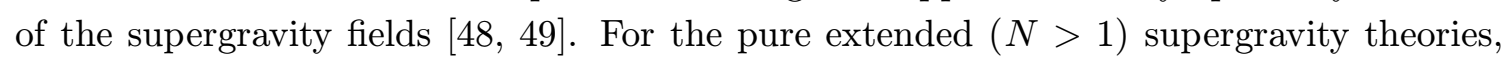
this phenomenon was analyzed in [50 terms in the algebra that are quadratic in the generators, in accord with the known form of the corresponding infinite-dimensional superconformal algebras. It should be interesting to extend this analysis to the propagating bulk fields described by the matter-coupled gauged supergravities of this paper.

In the spirit of the AdS/CFT correspondence the supergravity Lagrangians (6.5.5) obtained for the theories listed in table 25 allow the construction of the $n$-point correlation functions of a closed subset of chiral primary operators of the associated superconformal theories. To date, no concrete proposal for these $N=16$ boundary theories has been put forward - partly due to the lack of known brane configurations whose near horizon geometry would admit an isometry group related to any of the gauge groups in table $22_{-i}$ In contrast, the most prominent example of an $\mathrm{AdS}_{3} / \mathrm{CFT}_{2}$ correspondence, the D1-D5

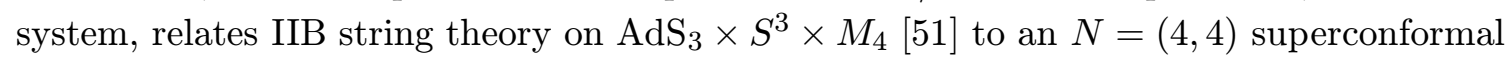
field theory described by a non-linear sigma model whose target space is a deformation of the symmetric orbifold $\left(M_{4}\right)^{n} / S_{n}\left[{ }_{6}^{6}\right]$. The corresponding low-energy effective supergravity

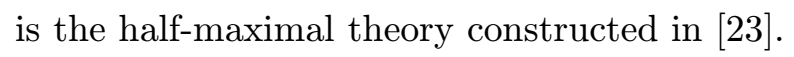

Gauged supergravities with non-semisimple gauge groups on the other hand make their appearance in the generalization of the AdS/CFT correspondence to so-called domain wall/QFT dualities, relating string theory on near-horizon Dp-brane geometries to $d=p+1$

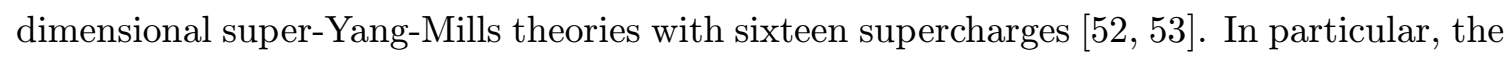
$N=16$ theory with gauge group $\mathrm{SO}(8) \ltimes \mathrm{T}_{28}$ describing the warped $\mathrm{AdS}_{3} \times S^{7}$ near-horizon

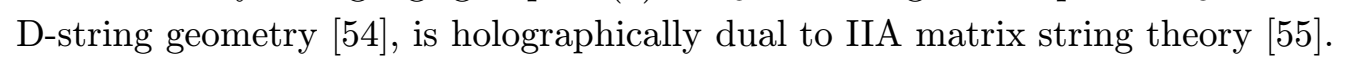

\section{Acknowledgments}

B. de Wit and H. Nicolai would like to thank the organizers for a very enjoyable meeting. We thank T. Fischbacher and I. Herger for collaboration on the results reported here. This work is partly supported by EU contracts HPRN-CT-2000-00122 and HPRN-CT-200000131, and by the INTAS contract 99-1-590. 


\section{References}

[1] B. de Wit, A. K. Tollstén, and H. Nicolai, Locally supersymmetric $D=3$ nonlinear sigma models, Nucl. Phys. B392 (1993) 3-38, [hep-th/9208074].

[2] H. Nicolai and H. Samtleben, Maximal gauged supergravity in three dimensions, Phys. Rev. Lett. 86 (2001) 1686-1689, [hep-th/0010076]; Compact and noncompact gauged maximal supergravities in three dimensions, JHEP 0104 (2001) 022, [hep-th/0103032].

[3] H. Nicolai and H. Samtleben, $N=8$ matter coupled $A d S_{3}$ supergravities, Phys. Lett. B514 (2001) 165-172, [hep-th/0106153].

[4] T. Fischbacher, H. Nicolai, and H. Samtleben, Non-semisimple and complex gaugings of $N=16$ supergravity, hep-th/0306276! to appear in Commun. Math. Phys.

[5] B. de Wit, I. Herger, and H. Samtleben, Gauged locally supersymmetric D $=3$ nonlinear sigma models, Nucl. Phys. B671 (2003) 175-216, [hep-th/0307006).

[6] O. Aharony, S. S. Gubser, J. Maldacena, H. Ooguri, and Y. Oz, Large N field theories, string theory and gravity, Phys. Rept. 323 (2000) 183-386, [hep-th/9̄ōji11i].

[7] M. Günaydin, L. J. Romans, and N. P. Warner, Compact and noncompact gauged supergravity theories in five-dimensions, Nucl. Phys. B272 (1986) 598-646.

[8] B. Julia, Application of supergravity to gravitation theories, in Unified field theories in more than 4 dimensions, eds. V.D. Sabbata and E. Schmutzer, World Scientific, Singapore 1983, 215

[9] N. Marcus and J. H. Schwarz, Three-dimensional supergravity theories, Nucl. Phys. B228 (1983) 145-162.

[10] E. Cremmer and B. Julia, The SO(8) Supergravity, Nucl. Phys. B159 (1979) 141.

[11] H. Nicolai and H. Samtleben, Chern-Simons vs. Yang-Mills gaugings in three dimensions, Nucl. Phys. B668 (2003) 167-178, [hep-th/0303213].

[12] T. Fischbacher, Some stationary points of gauged $N=16 D=3$ supergravity, Nucl. Phys. B638 (2002) 207-219, [hep-th/0201030i].

[13] T. Fischbacher, H. Nicolai, and H. Samtleben, Vacua of maximal gauged $D=3$ supergravities, Class. Quant. Grav. 19 (2002) 5297-5334, [hep-th/0207206].

[14] T. Fischbacher, Mapping the vacuum structure of gauged maximal supergravities: An application of high-performance symbolic algebra, hep-th/0305176.

[15] N.P. Warner, Some new extrema of the scalar potential of gauged $N=8$ supergravity, Phys. Lett. B128 (1983) 169

[16] B. de Wit and H. Nicolai, The parallelizing $S^{7}$ torsion in gauged $N=8$ supergravity, Nucl. Phys. B231 (1984) 506-532.

[17] C.M. Hull and N.P. Warner, The potentials of the gauged $N=8$ supergravity theories, Nucl. Phys. B253 (1985) 675

[18] C.M. Hull, The minimal couplings and scalar potentials of the gauged $N=8$ supergravities, Class. Quant. Grav. 2 (1985) 343

[19] K. Koepsell, H. Nicolai and H. Samtleben, An exceptional geometry for d = 11 supergravity?, Class. Quant. Grav. 17 (2000) 3689-3702, [hep-th/0006034]. 
[20] B. de Wit and H. Nicolai, Hidden symmetries, central charges and all that, Class. Quant. Grav. 18 (2001) 3095, [hep-th/0011239].

[21] E. Witten, String theory dynamics in various dimensions, Nucl. Phys. B443 (1995) 85-126, [hep-th/9503124.

[22] E. Witten, Strong coupling and the cosmological constant, Mod. Phys. Lett. A10 (1995) 2153, $[$ hep-th/9506-101].

[23] H. Nicolai and H. Samtleben, Kaluza-Klein supergravity on $A d S_{3} \times S^{3}$,JHEP 0309 (2003) 036 , [hep-th/0306202].

[24] E. Witten, Quantization of Chern-Simons gauge theory with complex gauge group, Commun. Math. Phys. 137 (1991) 29-66.

[25] R. Kashaev and N. Reshetikhin, Invariants of tangles with flat connections in their complements. I. Invariants and holonomy R-matrices, math.AT/020221 i]

[26] S. Gukov, Three-dimensional quantum gravity, Chern-Simons theory, and the A-polynomial, [hep-th/0306165is.

[27] J. Gegenberg, S. Vaidya, and J. F. Vazquez-Poritz, Thurston geometries from eleven dimensions, Class. Quant. Grav. 19 (2002) L199-L204, [hep-th/0205276].

[28] J. Gegenberg and G. Kunstatter, Using 3d stringy gravity to understand the Thurston conjecture, hep-th/0306279i.

[29] G. Perelman, The entropy formula for the Ricci flow and its geometric applications,

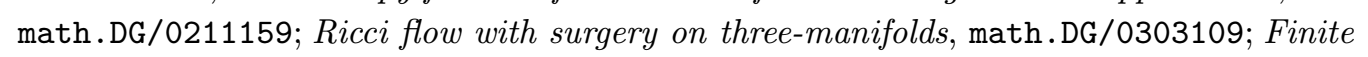
extinction time for the solutions to the Ricci flow on certain three-manifolds, math.DG/0307245.

[30] S. Deser, R. Jackiw and G. 't Hooft, Three-dimensional Einstein gravity: Dynamics of flat space Ann. Phys. 152 (1984) 220

[31] S. Deser and R. Jackiw, Three-Dimensional Cosmological Gravity: Dynamics Of Constant Curvature, Annals Phys. 153 (1984) 405.

[32] A. Achúcarro and P. K. Townsend, A Chern-Simons action for three-dimensional anti-de Sitter supergravity theories, Phys. Lett. B180 (1986) 89-92.

[33] M. Cvetič, H. Lu, and C. N. Pope, Consistent Kaluza-Klein sphere reductions, Phys. Rev. D62 (2000) 064028, [hep-th/0003286i.

[34] H. Lu, C. N. Pope, and E. Sezgin, SU(2) reduction of six-dimensional (1,0) supergravity, Nucl. Phys. B668 (2003) 237-257, [hep-th/0212323]; Yang-Mills-Chern-Simons supergravity, hep-th/0305242i

[35] S. Deser and Z. Yang, A Remark On The Higgs Effect In Presence Of Chern-Simons Terms, Mod. Phys. Lett. A4 (1989) 2123.

[36] B. de Wit and H. Nicolai, N=8 supergravity, Nucl. Phys. B208 (1982) 323-364.

[37] J. M. Izquierdo and P. K. Townsend, Supersymmetric space-times in (2+1) AdS supergravity models, Class. Quant. Grav. 12 (1995) 895-924, [gr-qc/9501018'].

[38] N. S. Deger, A. Kaya, E. Sezgin, and P. Sundell, Matter coupled $A d S_{3}$ supergravities and their black strings, Nucl. Phys. B573 (2000) 275-290, hep-th/9908089.]. 
[39] M. Abou-Zeid and H. Samtleben, Chern-Simons vortices in supergravity, Phys. Rev. D65 (2002) 085016, hep-th/0112035i.

[40] M. Berg, M. Haack, and H. Samtleben, Calabi-Yau fourfolds with flux and supersymmetry breaking, JHEP 04 (2003) 046, [hep-th/0212255.

[41] B. de Wit, Supergravity, in Unity from Duality: Gravity, Gauge Theory and Strings (C. Bachas, M. Douglas, A. Bilal, N. Nekrasov, and F. David, eds.), Springer Verlag, 2003. 'hep-th/02 12245 '.

[42] C. M. Hull, Noncompact gaugings of $N=8$ supergravity, Phys. Lett. B142 (1984) 39-41; More gaugings of $N=8$ supergravity, Phys. Lett. B148 (1984) 297-300; A new gauging of $N=8$ supergravity, Phys. Rev. D30 (1984) 760 .

[43] L. Andrianopoli, F. Cordaro, P. Fré, and L. Gualtieri, Non-semisimple gaugings of $D=5$ $N=8$ supergravity and FDAs, Class. Quantum Grav. 18 (2001) 395-413, [hep-th/0009048i].

[44] L. Andrianopoli, R. D'Auria, S. Ferrara, and M. A. Lledó, Gauging of flat groups in four dimensional supergravity, JHEP 07 (2002) 010, |hep-th/0203206.].

[45] C. M. Hull, New gauged $N=8, D=4$ supergravities, Class. Quant. Grav. 20 (2003) 5407-5424, [hep-th/0204156.

[46] B. de Wit, H. Samtleben, and M. Trigiante, On Lagrangians and gaugings of maximal supergravities, Nucl. Phys. B655 (2003) 93-126, [hep-th/0212239i; Maximal supergravity from IIB flux compactifications, Phys. Lett. B583 (2004) 338-346, [hep-th/0311224].

[47] E. S. Fradkin and V. Y. Linetsky, Results of the classification of superconformal algebras in two-dimensions, Phys. Lett. B282 (1992) 352, [hep-th/9203045]; Classification of superconformal and quasisuperconformal algebras in two-dimensions, Phys. Lett. B291 (1992) 71.

[48] J. D. Brown and M. Henneaux, Central charges in the canonical realization of asymptotic symmetries: An example from three-dimensional gravity, Commun. Math. Phys. 104 (1986) 207.

[49] M. Banados, K. Bautier, O. Coussaert, M. Henneaux and M. Ortiz, Anti-de Sitter/CFT correspondence in three-dimensional supergravity, Phys. Rev. D58 (1998) 085020, [hep-th/9805165i.

[50] M. Henneaux, L. Maoz and A. Schwimmer, Asymptotic dynamics and asymptotic symmetries of three-dimensional extended AdS supergravity, Annals Phys. 282 (2000) 31, [hep-th/9910013.

[51] A. Giveon, D. Kutasov and N. Seiberg, Comments on string theory on $A d S_{3}$, Adv. Theor. Math. Phys. 2 (1998) 733, [hep-th/9806194]; D. Kutasov and N. Seiberg, More comments on string theory on $A d S_{3}, J H E P \mathbf{9 9 0 4}(1999) 008$, [hep-th/9903219].

[52] N. Itzhaki, J. M. Maldacena, J. Sonnenschein and S. Yankielowicz, Supergravity and the large $N$ limit of theories with sixteen supercharges, Phys. Rev. D58 (1998) 046004, $[$ hep-th/9802042i].

[53] H. J. Boonstra, K. Skenderis and P. K. Townsend, The domain wall/QFT correspondence, JHEP 9901 (1999) 003, [hep-th/9807137].

[54] J. F. Morales and H. Samtleben, Supergravity duals of matrix string theory, JHEP 0208 (2002) 042 , [hep-th/0206247it. 
[55] R. Dijkgraaf, E. Verlinde and H. Verlinde, Matrix string theory, Nucl. Phys. B500 (1997) 43, [hep-th/9703030i. 